\title{
ALMA finds dew drops in the dusty spider's web
}

\author{
Bitten Gullberg ${ }^{1,3,4}$, Matthew D. Lehnert ${ }^{2}$, Carlos De Breuck ${ }^{1}$, Steve Branchu ${ }^{1,5}$, Helmut Dannerbauer ${ }^{6}$, \\ Guillaume Drouart ${ }^{7}$, Bjorn Emonts ${ }^{8}$, Pierre Guillard ${ }^{2}$, Nina Hatch ${ }^{9}$, Nicole P. H. Nesvadba ${ }^{10}$, Alain Omont ${ }^{2}$, \\ Nick Seymour ${ }^{11}$, and Joël Vernet ${ }^{1}$ \\ ${ }^{1}$ European Southern Observatory, Karl-Schwarzschild-Str. 2, 85748 Garching, Germany \\ e-mail: bitten.gullberg@durham.ac.uk \\ 2 Institut d'Astrophysique de Paris, UMR 7095, CNRS, Université Pierre et Marie Curie, 98bis boulevard Arago, \\ 75014 Paris, France \\ 3 Max-Planck-Institut für Extraterrestrische Physik, Giessenbachstraße 1, 85748 Garching, Germany \\ ${ }^{4}$ Centre for Extragalactic Astronomy, Department of Physics, Durham University, South Road, Durham DH1 3LE, UK \\ 5 Université de Bordeaux, LAB, UMR 5804, 33270 Floirac, France \\ ${ }^{6}$ Universität Wien, Institut für Astrophysik, Türkenschanzstraße 17, 1180 Wien, Austria \\ 7 Department of Earth and Space Science, Chalmers University of Technology, Onsala Space Observatory, 43992 Onsala, Sweden \\ 8 Centro de Astrobiología (INTA-CSIC), Ctra de Torrejón a Ajalvir, km 4, 28850 Torrejń de Ardoz, Madrid, Spain \\ 9 School of Physics and Astronomy, University of Nottingham, University Park, Nottingham NG7 2RD, UK \\ 10 Institut d'Astrophysique Spatiale, CNRS, Université Paris-Sud, Bât. 120-121, 91405 Orsay, France \\ 11 International Center for Radio Astronomy Research, Curtin University, GPO Box U1987, Perth, WA 6845, Australia
}

Received 26 October 2015 / Accepted 10 February 2016

\begin{abstract}
We present $0 \prime .5$ resolution ALMA detections of the observed $246 \mathrm{GHz}$ continuum, [CI] ${ }^{3} \mathrm{P}_{2} \rightarrow{ }^{3} \mathrm{P}_{1}$ fine structure line ([CI]2-1), $\mathrm{CO}(7-6)$, and $\mathrm{H}_{2} \mathrm{O}$ lines in the $z=2.161$ radio galaxy MRC1138-262, the so-called Spiderweb galaxy. We detect strong [CI]2-1 emission both at the position of the radio core, and in a second component $\sim 4 \mathrm{kpc}$ away from it. The $1100 \mathrm{~km} \mathrm{~s}^{-1}$ broad [CI] $2-1$ line in this latter component, combined with its $\mathrm{H}_{2}$ mass of $1.6 \times 10^{10} M_{\odot}$, implies that this emission must come from a compact region $<60 \mathrm{pc}$, possibly containing a second active galactic nucleus (AGN). The combined $\mathrm{H}_{2}$ mass derived for both objects, using the $[\mathrm{CI}] 2-1 \mathrm{emis}-$ sion, is $3.3 \times 10^{10} M_{\odot}$. The total $\mathrm{CO}(7-6) /[\mathrm{CI}] 2-1$ line flux ratio of 0.2 suggests a low excitation molecular gas reservoir and/or enhanced atomic carbon in cosmic ray dominated regions. We detect spatially-resolved $\mathrm{H}_{2} \mathrm{O} 22_{11}-2_{02}$ emission - for the first time in a high- $z$ unlensed galaxy - near the outer radio lobe to the east, and near the bend of the radio jet to the west of the radio galaxy. No underlying $246 \mathrm{GHz}$ continuum emission is seen at either position. We suggest that the $\mathrm{H}_{2} \mathrm{O}$ emission is excited in the cooling region behind slow $\left(10-40 \mathrm{~km} \mathrm{~s}^{-1}\right)$ shocks in dense molecular gas $\left(10^{3-5} \mathrm{~cm}^{-3}\right)$. The extended water emission is likely evidence of the radio jet's impact on cooling and forming molecules in the post-shocked gas in the halo and inter-cluster gas, similar to what is seen in low $z$ clusters and other high- $z$ radio galaxies. These observations imply that the passage of the radio jet in the interstellar and inter-cluster medium not only heats gas to high temperatures, as is commonly assumed or found in simulations, but also induces cooling and dissipation, which can lead to substantial amounts of cold dense molecular gas. The formation of molecules and strong dissipation in the halo gas of MRC1138-262 may explain both the extended diffuse molecular gas and the young stars observed around MRC1138-262.
\end{abstract}

Key words. galaxies: evolution - galaxies: high-redshift - galaxies: active - galaxies: ISM - Galaxy: halo

\section{Introduction}

The high- $z$ radio galaxy $(\mathrm{H} z \mathrm{RG}) \mathrm{MRC} 1138-262$ at $z=2.161$ is one of the best studied HzRG (e.g. Pentericci et al. 1997, 1998, 2000; Carilli et al. 1997, 2002; Stevens et al. 2003; Kurk et al. 2004b,a; Greve et al. 2006; Miley et al. 2006; Hatch et al. 2008, 2009; Humphrey et al. 2008; Kuiper et al. 2011; Ogle et al. 2012; Seymour et al. 2012), with a well sampled spectral energy distribution (SED) that covers from radio to X-ray. Its radio morphology is typical of distant radio galaxies with a string of radio bright knots along the radio jet that extends to the west and a single lobe to the east of the central radio core (Carilli et al. 1997). The radio core has an "ultra steep" spectral index of $\alpha=-1.2$,

* The reduced data cubes are only available at the CDS via anonymous ftp to cdsarc.u-strasbg. fr $(130.79 .128 .5)$ or via http: //cdsarc.u-strasbg.fr/viz-bin/qcat?]/A+A/591/A73 and the spectral indices of the radio knots systematically steepen with increasing distance from the core (Pentericci et al. 1997).

The radio source is embedded in an environment that is overdense in galaxies, over scales of hundreds of kpc (Pentericci et al. 1998; Miley et al. 2006) to Mpc scales (Pentericci et al. 2002; Kurk et al. 2004b,a; Dannerbauer et al. 2014). Many of these galaxies are clumpy and star-forming (Pentericci et al. 1998; Miley et al. 2006; Dannerbauer et al. 2014). Hatch et al. (2009) predict that most of the satellite galaxies within $150 \mathrm{kpc}$ will merge with the central $\mathrm{H} z \mathrm{RG}$ and that the final merger galaxy will contain very little gas owing to the high star formation rate (SFR) of the satellite galaxies. X-ray observations of MRC1138262 are inconclusive about the existence of an extended X-ray atmosphere (cf. Carilli et al. 2002; Pentericci et al. 2000, the extended X-ray emission could be due to inverse Compton or shocks generated by the passage of the radio jets). Pentericci et al. (2000), who favour the existence of a large thermal hot 
X-ray emitting atmosphere, conclude that MRC1138-262 has many of the necessary ingredients of a forming galaxy cluster, i.e. an irregular velocity distribution of the Ly $\alpha$ emitting galaxies, an over-density of galaxies, a massive central galaxy, and a hot X-ray halo. Owing to the large number of companion galaxies surrounding the $\mathrm{HzRG}$ being analogous to "flies caught in a spiders web", MRC1138-262 was dubbed the Spiderweb galaxy (Miley et al. 2006).

Multi-wavelength photometry, including the infrared continuum emission (Stevens et al. 2003; Greve et al. 2006; De Breuck et al. 2010), implies an extremely high SFR in the Spiderweb galaxy. Fitting an active galactic nucleus (AGN) and starburst component SED to the mid- to far-IR SED, Seymour et al. (2012) find a SFR for the starburst component of $1390 \pm 150 M_{\odot} \mathrm{yr}^{-1}$ (see also Ogle et al. 2012). This should be compared with the rest-frame UV estimates of only a couple $100 M_{\odot} \mathrm{yr}^{-1}$ (even with an extinction correction) by Hatch et al. (2008), who emphasise how deeply embedded the majority of the intense star formation is in MRC1138-262. Although the star formation is intense, the characteristics of the vigorous outflow $\left(\dot{M} \gtrsim 400 M_{\odot} \mathrm{yr}^{-1}\right)$ that is observed in the optical emission line gas suggest that this is predominately driven by the radio jet (Nesvadba et al. 2006).

Beyond the companion galaxies of the $\mathrm{HzRG}$, the diffuse stellar and gaseous environment of MRC1138-262 on larger scales is also fascinatingly complex. MRC1138-262 has a significant amount of diffuse UV intergalactic light (IGL) within $60 \mathrm{kpc}$ of the radio galaxy, which indicates ongoing star formation in the circumgalactic environment (Hatch et al. 2008). This diffuse light is embedded in a large $(\sim 100 \mathrm{kpc}$ in diameter $)$ Ly $\alpha$ emitting halo (Pentericci et al. 1997). Using semi-analytical models, Hatch et al. (2008) ruled out the possibility that the observed circumgalactic light originates from unresolved, lowmass satellite galaxies. Spectra extracted at the position of the central HzRG, of a nearby galaxy and the IGL, show Ly $\alpha$ emission lines with absorption troughs superimposed, which suggests the presence of warm neutral gas mixed with the ionised gas that surrounds the HzRG (Pentericci et al. 1997; Hatch et al. 2008). This leads to the conclusion that the large Ly $\alpha$ halo emission is powered not only by the extended and diffuse star formation (Pentericci et al. 1998; Miley et al. 2006; Hatch et al. 2008) but also by AGN photoionisation and shock heating (Nesvadba et al. 2006).

The influence of the radio jet from the AGN is seen in the VLA observations, which reveal a bend in the western string of clumps detected $\sim 20 \mathrm{kpc}$ from the core towards the southwest. Ly $\alpha$ line emitting gas has a bright spot associated with this bend and the two hotspots, implying the presence of a massive cloud of gas deflecting the radio jet and causing these features (Pentericci et al. 1997; Lonsdale \& Barthel 1986). Several different models exist for how gas might deflect radio jets, such as the jet drilling into a gas cloud where it blows a bubble in the hot plasma (Lonsdale \& Barthel 1986) or through the counter pressure generated by oblique reverse shocks in the cloud generated by a jet-cloud interaction (Bicknell et al. 1998). Given this situation, Pentericci et al. (1997) argue that the relation between the radio emitting knots in the jet and the high surface brightness Ly $\alpha$ halo emission, especially where the eastern jet bends, suggest an interaction between the jet and the ambient gas in the halo of MRC1138-262. However, the origin of the Ly $\alpha$ emitting gas reservoir is still uncertain.

This high an SFR of the $\mathrm{HzRG}$ means that a significant molecular gas reservoir fuelling the star formation must be present. Emonts et al. (2013) probe the diffuse extended molecular gas reservoir using the $\mathrm{CO}(1-0)$ line. They find that there is approximately $6 \times 10^{10} M_{\odot}$ of cold $\mathrm{H}_{2}$ gas over a scale of $10 \mathrm{~s}$ of $\mathrm{kpc}$, which surrounds the $\mathrm{HzRG}$ (also Emonts et al., in prep.). The kinematics of the cold molecular gas is relatively quiescent (Emonts et al., in prep.). More surprisingly, Ogle et al. (2012) detect the 0-0 S(3) rotational line of $\mathrm{H}_{2}$ in MRC1138-262. The strength of the line, allowing for a range of plausible excitation temperatures of $\mathrm{H}_{2}$, imply warm $(T>300 \mathrm{~K}) \mathrm{H}_{2}$ masses of order $10^{7}$ to $10^{9} M_{\odot}$. While the large Spitzer beam does not allow the $\mathrm{H}_{2}$ emission to be spatially resolved, a plausible interpretation of the relatively large mass of warm $\mathrm{H}_{2}$ gas in MRC1138262 is that a fraction of the jet energy is being dissipated as supersonic turbulence and shocks in the dense gas in the immediate environment of the AGN.

Although the optically thick $\mathrm{CO}(1-0)$ emission line is a good tracer of the diffuse molecular gas, the emission lines from neutral carbon $([\mathrm{CI}])$ are arguably even better. The $[\mathrm{CI}]$ lines have critical densities similar to the low- $J$ CO lines, meaning that they probe the same phases of the molecular gas. As they are both optically thin, they therefore probe higher column densities than CO (Papadopoulos et al. 2004). While the [CI] lines are good tracers of the diffuse molecular gas, they are poor tracers of the very dense star-forming gas. Molecular lines from, for example, $\mathrm{H}_{2} \mathrm{O}, \mathrm{HCN}$, and $\mathrm{CS}$ have a much higher critical density, and therefore probe the dense molecular star-forming phase. Omont et al. (2013) find a relation between the far-infrared (FIR) and $\mathrm{H}_{2} \mathrm{O}$ luminosities for a sample of high- $z$ starburst galaxies. The $\mathrm{H}_{2} \mathrm{O}$ detections for this sample are all associated with underlying FIR emission, implying that the $\mathrm{H}_{2} \mathrm{O}$ emission traces star-forming regions. However, the $\mathrm{H}_{2} \mathrm{O}$ molecules can also be excited in the dissipation of supersonic turbulence in molecular gas or by slow shocks (e.g. Flower \& Pineau Des Forêts 2010). In the case of purely shock-excited $\mathrm{H}_{2} \mathrm{O}$, it is unlikely that underlying FIR emission would be detected in regions of strong $\mathrm{H}_{2} \mathrm{O}$ emission (e.g. Goicoechea et al. 2015).

Motivated to determine the energy source and distribution of the strong dissipation as possibly observed through $\mathrm{H}_{2}$ emission and to determine the state of the molecular gas in MRC1138262, we proposed for ALMA observations in Cycle 1. In this paper, we present our results for the observed $246 \mathrm{GHz}$ continuum emission (rest-frame $\sim 740 \mathrm{GHz}$ ), $\mathrm{H}_{2} \mathrm{O} 2_{11}-2_{02}$ transition (at $v_{\text {rest }}=752.03 \mathrm{GHz}$, which hereafter is often referred to as $\mathrm{H}_{2} \mathrm{O}$ ), $[\mathrm{CI}]{ }^{3} \mathrm{P}_{2} \rightarrow{ }^{3} \mathrm{P}_{1}$ fine structure emission line (at $v_{\text {rest }}=809.34 \mathrm{GHz}$, which hereafter is referred to as [CI]2-1), and $\mathrm{CO}(7-6)$ emission line observations towards the Spiderweb galaxy. We find strong $246 \mathrm{GHz}$ continuum emission at the position of the HzRG. Near both radio lobes, we detect emission from $\mathrm{H}_{2} \mathrm{O}$, the first spatially resolved detection of $\mathrm{H}_{2} \mathrm{O}$ in a high- $z$ unlensed galaxy. We also detect strong [CI]2-1 emission that is blended with weak $\mathrm{CO}(7-6)$ emission at the position of the $\mathrm{H}_{z} \mathrm{RG}$. In Sect. 2, we present our Atacama Large Millimeter/submillimeter Array (ALMA) submm observations. The results of these observations are given in Sect. 3. We analyse and discuss them in Sect. 4 and summarise our conclusions in Sect. 5. We assume $H_{0}=73 \mathrm{~km} \mathrm{~s}^{-1} \mathrm{Mpc}^{-1}, \Omega_{\mathrm{M}}=0.27$, and $\Omega_{\Lambda}=0.73$, which implies a scale of $8.172 \mathrm{kpc} /{ }^{\prime \prime}$ at $z=2.161$.

\section{Observations}

\subsection{ALMA observations}

The ALMA cycle 1 Band 6 observations were carried out on 2014 April 27 for 49 min on-source time with 36 working antennas. The four $1.875 \mathrm{GHz}$ spectral windows were tuned to cover the frequency ranges $237.3-240.9 \mathrm{GHz}$ and 
252.6-256.7 GHz. We used the supplied Common Astronomy Software Applications (CASA) calibration script to produce the data cube, continuum map, and moment- 0 maps. The quasar J1146-2859 was used as a bandpass calibrator, and the UV range was well covered within $400 \mathrm{k} \lambda$.

We made a natural weighted map (Briggs robust parameter of 2), which exhibits the highest signal-to-noise ratio $(\mathrm{S} / \mathrm{N})$, at the expense of a lower spatial resolution. The frequency range between $254.9-265.7 \mathrm{GHz}$ (i.e. half of the upper side band) is dominated by strong [CI]2-1 and $\mathrm{CO}(7-6)$ line emission and is therefore not included in the continuum map. This results in a continuum map with a synthesised beam of 0 ". $69 \times 0$ ".44 with PA $89.3^{\circ}$ and a root mean square (rms) of $50 \mu \mathrm{Jy} /$ beam. We corrected the continuum map for the primary beam of $26^{\prime \prime} 3$.

The $\mathrm{H}_{2} \mathrm{O}$ emission is spatially offset from the continuum emission and a continuum subtraction is therefore not required, as it would only, unnecessarily, add noise. The $\mathrm{H}_{2} \mathrm{O}$ observations are therefore not continuum-subtracted. The [CI]2-1 and $\mathrm{CO}(7-6)$ emission lines are within the frequency range at $z=2.1606$, and we subtract the continuum in the UV-plane by fitting a first order polynomial to the line free channels. The

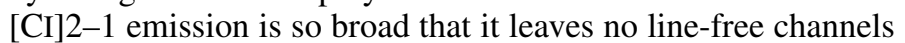
in spectral window 0 . We therefore fit the continuum to spectral window 1, which shows no signs of line emission. We bin the [CI]2-1 line data to $20 \mathrm{~km} \mathrm{~s}^{-1}$, which has an rms of $0.5 \mathrm{mJy}$ and the $\mathrm{H}_{2} \mathrm{O}$ line data to $60 \mathrm{~km} \mathrm{~s}^{-1}$, which has an rms of $0.3 \mathrm{mJy}$. We primary beam-correct the $\mathrm{H}_{2} \mathrm{O}$ line data, since we find $\mathrm{H}_{2} \mathrm{O}$ emission $\sim 6$ " 5 from the phase centre, where the sensitivity is at $87 \%$.

\section{Results}

The Spiderweb galaxy is detected in both $246 \mathrm{GHz}$ continuum and $[\mathrm{CI}] 2-1, \mathrm{CO}(7-6)$ and $\mathrm{H}_{2} \mathrm{O}$ line emission. Figure 1 shows an overview of the spatial distribution of the different components. The strong [CI]2-1 line emission is seen in two components that are separated by $0 . \prime 5$. The $[\mathrm{CI}] 2-1$ component 1 is centred at the position of the $\mathrm{HzRG}$ (shown with a blue ellipse in Fig. 1) and the $[\mathrm{CI}] 2-1$ component 2 is located $0 \prime \prime 5$ to the south-east (shown with a red ellipse in Fig. 1). The $246 \mathrm{GHz}$ continuum emission peaks at the position of the $\mathrm{H} z \mathrm{RG}$ (shown with green ellipses in Fig. 1), but shows an extension in the direction of the [CI]2-1 component 2. Emission from $\mathrm{H}_{2} \mathrm{O}$ is also detected south-west of the $\mathrm{H}_{z} \mathrm{RG}$ (shown with the orange ellipse, south of the $246 \mathrm{GHz}$ continuum component in Fig. 1), which is co-spatial with the bend in the radio jet (Carilli et al. 1997). $\mathrm{H}_{2} \mathrm{O}$ emission is detected east of the $\mathrm{HzRG}$ - west of knot $\mathrm{A}$ in the radio jet (shown with an orange ellipse to the left, in Fig. 1). We now discuss each of the components separately. Table 2 lists the derived line parameters. Throughout, we adopt the $[\mathrm{CI}] 2-1$ emission at the position of the radio core as the systemic redshift $z=2.1606$.

\subsection{Continuum emission}

The continuum map contains bright $246 \mathrm{GHz}$ continuum emission at the position of the $\mathrm{H}_{z} \mathrm{RG}, \sim 20 \mathrm{kpc}$ to the west and tentative emission to the north (see Fig. 1).

\subsubsection{The HzRG}

The $246 \mathrm{GHz}$ continuum emission at the position of the $\mathrm{Hz} \mathrm{RG}$ shows an east-west elongation, in the same orientation as the radio source (see Fig. 1). A similar orientation was seen in the

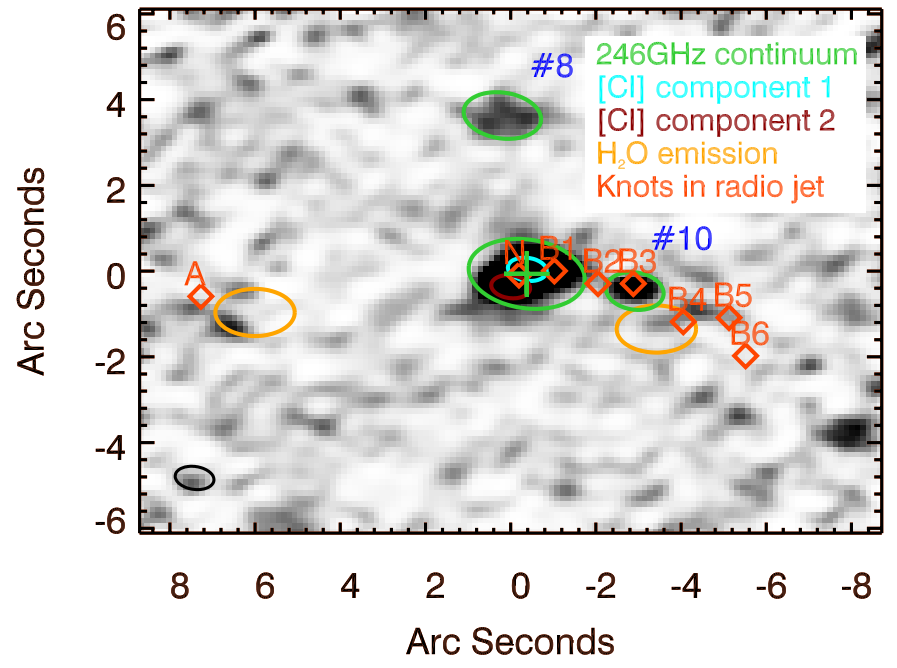

Fig. 1. Overview of the spatial distribution of the detected components. The naturally weighted $246 \mathrm{GHz}$ continuum map is in greyscale and the two [CI]2-1 components, 1 and 2, are shown with blue and red ellipses, respectively. The two $\mathrm{H}_{2} \mathrm{O}$ detections are shown with orange ellipses, and the $246 \mathrm{GHz}$ continuum components shown with green ellipses. The sizes of the ellipses represent the extractions used for the photometry. The knots in the radio jet are indicated with red-orange diamonds and labeled according to Pentericci et al. (1997). The numbers correspond to the numbering in Kuiper et al. (2011). The ALMA beam is shown as a black ellipse in the lower left corner.

SCUBA and LABOCA maps (Stevens et al. 2003; Dannerbauer et al. 2014), but those extensions were on a much larger scale than the separation seen in Fig. 1. Although the continuum only has one peak, the elongation suggests a two component system, similar to that seen in the $[\mathrm{CI}] 2-1$ line emission (see Sect. 3.2.1). Using the peak positions from component 1 and 2 in the $[\mathrm{CI}] 2-1$ moment-0 maps, we fit a double 2D Gaussian profile to the continuum by fixing the centres of the Gaussians at the peak positions of the $[\mathrm{CI}] 2-1$ emission line. This results in a continuum ratio for the two components of $\sim 5$. Integrating the fitted double 2D Gaussian yields the flux density of $1.78 \pm 0.29 \mathrm{mJy}$ (see Table 1). This emission is probably dominated by thermal dust emission, although we warn that a linear extrapolation of the $S(8.2 \mathrm{GHz})=1.88 \mathrm{mJy}$ (Pentericci et al. 1997) and the $S(36.5 \mathrm{GHz})=1.02 \mathrm{mJy}$ (Emonts et al., in prep.) for the core predicts a synchrotron contribution of $0.47 \mathrm{mJy}$ at $246 \mathrm{GHz}$. Any star formation parameters derived directly from the $246 \mathrm{GHz}$ should therefore be considered as an upper limit.

\subsubsection{The companion sources}

We detect $246 \mathrm{GHz}$ continuum emission from a bright companion $\sim 20 \mathrm{kpc}$ to the west of the $\mathrm{H}_{z} \mathrm{RG}$ (see Fig. 1). This companion is associated with knot B3 in the radio jet (Carilli et al. 1997; Pentericci et al. 1997, see Fig. 1) and a small group of galaxies (denoted D by Pentericci et al. 1997, and \#10 by Kuiper et al. 2011, see Fig. 2). This is also the position of a high surface brightness region of Ly $\alpha$ emission (Pentericci et al. 1997). This companion is also seen in, for example, HST F814W imaging (Miley et al. 2006) and at other optical wavelengths, e.g. $R$-band (Pentericci et al. 1997). Companion \#10 is co-spatial with companion D in Pentericci et al. (1997) at RA = 11:40:48.14, Dec $=$ -26.29 .09 .2 , and is offset by only 0 ' 8 to the Ly $\alpha$ emitter \#491 from Kurk et al. (2004b). This Ly $\alpha$ emitter at RA = 11:40:48.2, Dec $=-26.29 .09 .5$ (shown with the purple circle in Figs. 2) is 
Table 1. Peak positions and $246 \mathrm{GHz}$ continuum flux of the $\mathrm{HzRG}$ and companion \#8 and \#10.

\begin{tabular}{|c|c|c|c|c|}
\hline \multirow[t]{2}{*}{ Component } & \multicolumn{2}{|c|}{ Position } & \multirow{2}{*}{$\begin{array}{c}S_{246 \mathrm{GHz}} \\
\mathrm{mJy}\end{array}$} & \multirow[t]{2}{*}{$z$} \\
\hline & RA & Dec & & \\
\hline $\mathrm{Hz} z \mathrm{RG}$ & $11: 40: 48.34$ & $-26: 29: 08.656$ & $1.78 \pm 0.29$ & $2.1606 \pm 0.0041$ \\
\hline Companion \#8 & $11: 40: 48.38$ & $-26: 29: 04.940$ & $<0.25$ & 2.1437 \\
\hline Companion \#10 & $11: 40: 48.15$ & $-26: 29: 09.490$ & $0.19 \pm 0.01$ & 2.1446 \\
\hline Water (west) & $11: 40: 48.82$ & $-26: 29: 09.58$ & $<0.2$ & \\
\hline Water (east) & $11: 40: 48.12$ & $-26: 29: 09.97$ & $<0.2$ & \\
\hline
\end{tabular}

Notes. The continuum fluxes are calculated by integrating under the fitted double 2D Gaussian profile. Companion \#8 is a tentative detection and therefore an upper limit. We take the $3 \sigma$ upper limit of the $246 \mathrm{GHz}$ continuum emission at the positions of the $\mathrm{H}_{2} \mathrm{O}$ emission to the west and east to be three times the rms.

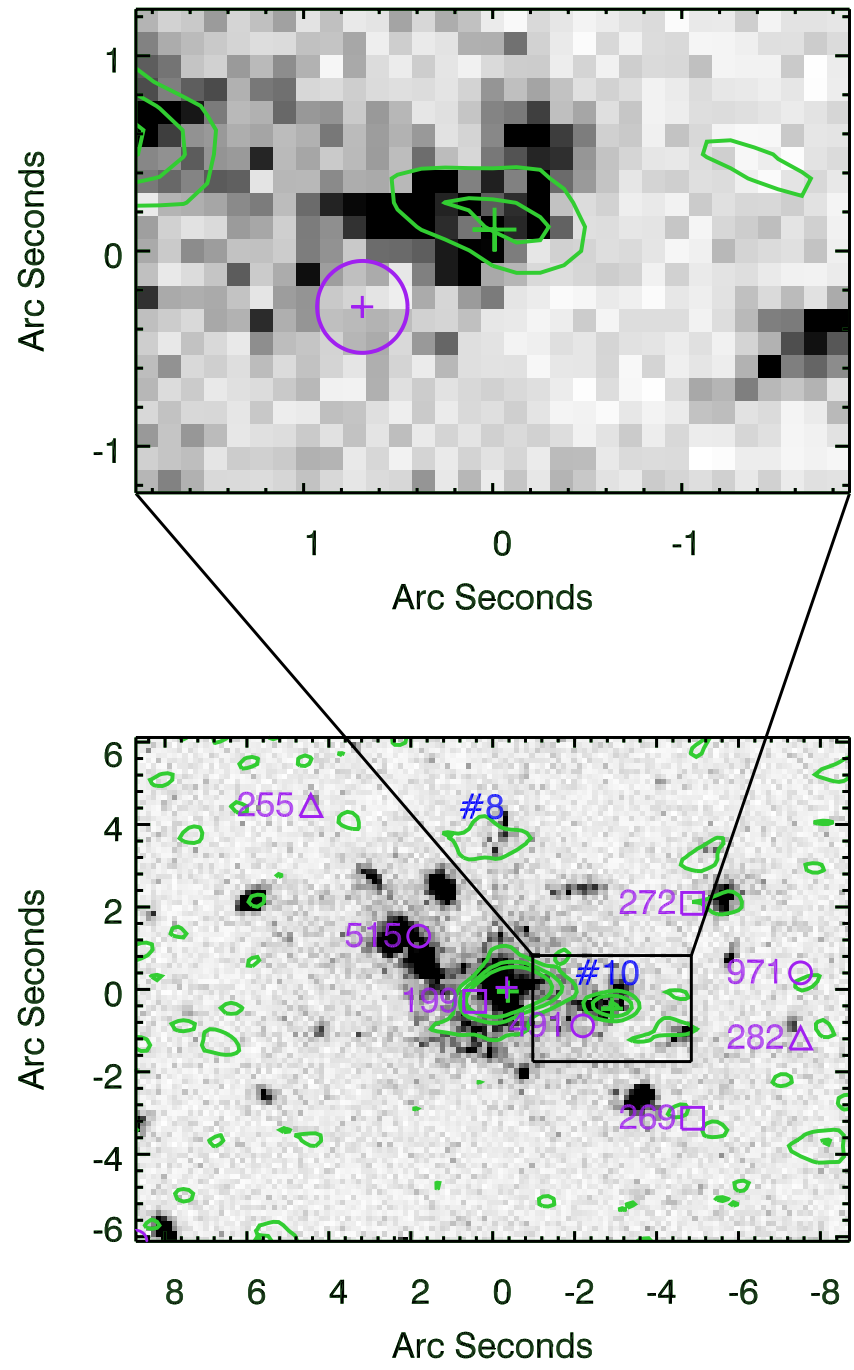

Fig. 2. Bottom panel: HST F814W image (Hatch et al. 2008) in greyscale overlaid with the naturally weighted $246 \mathrm{GHz}$ continuum map in green contours with levels of $1.5 \sigma, 3 \sigma$, and $5 \sigma$. In the naturally weighted continuum map, we detect emission from companion \#10 (and tentatively from \#8) of Kuiper et al. (2011). The purple cross indicate the position of the nearby Ly $\alpha$ emitter at the position of the $\mathrm{H} z \mathrm{RG}$. The purple circles indicate the positions of Ly $\alpha$ emitters, the squares show the position of $\mathrm{H} \alpha$ and the triangles extremely red objects within the Ly $\alpha$ halo of MRC1138-262. The Ly $\alpha$ emitter \#491 is offset by $00^{\prime \prime} 8$ from the companion source seen in the $246 \mathrm{GHz}$ continuum emission west of the HzRG. Top panel: zoom in of the region around the companion sources. The purple circle shows the position of the $\mathrm{H} \alpha$ emitter \#491 (Kurk et al. 2004b), which is close to the companion source seen in dust continuum emission. inside the Ly $\alpha$ halo of the Spiderweb galaxy (Kurk et al. 2004b), and so are three other Ly $\alpha$ emitters, three $\mathrm{H} \alpha$ emitters, and two extremely red objects (ERO, also indicated in Fig. 2). Fitting a 2D Gaussian profile to companion \#10, we find a flux density of $0.19 \pm 0.01 \mathrm{mJy}$ (see Table 1$)$.

To examine the nature of the $246 \mathrm{GHz}$ emission of companion \#10, we compared the flux density in the spectral windows that were not contaminated by line emission. The expected ratio between 238.28 and $253.48 \mathrm{GHz}$ (i.e. the lower and upper side bands: LSB and USB) from thermal blackbody radiation at $40 \mathrm{~K}$ is 0.80 , while the observed ratio is $1.19 \pm 0.22$. The Decreasing spectral slope with increasing frequency of \#10 is more consistent with a synchrotron rather than a thermal dust origin. Indeed, a straight extrapolation of the $S_{8.2 \mathrm{GHz}}=5.9 \mathrm{mJy}$ (Pentericci et al. 1997) and the $S_{36.5 \mathrm{GHz}}=1.8 \mathrm{mJy}$ (Emonts et al., in prep.) implies $S_{239 \mathrm{GHz}}=0.4 \mathrm{mJy}$. Our observed $0.2 \mathrm{mJy}$ is therefore fully consistent with synchrotron emission, and even allows for the expected spectral steepening at high frequencies. Thus synchrotron-dominated submm emission is consistent with companion \#10 having very blue UV colours with no signs of a Balmer break or significant extinction (Hatch et al. 2009). On the other hand, it could also be that the most obscured regions are not visible in the optical images, but only the bluest, least obscured regions are. Higher resolution dust continuum and a broad submillimetre wavelength coverage can test these hypotheses.

\section{2. [Cl]2-1 and $\mathrm{CO}(7-6)$ line emission}

\subsubsection{The HzRG}

Strong emission from the $[\mathrm{CI}]{ }^{3} \mathrm{P}_{2} \rightarrow{ }^{3} \mathrm{P}_{1}$ fine structure emission line is detected at the position of the $\mathrm{H}_{z} \mathrm{RG}$. The [CI $] 2-1$ emission line has a double peaked velocity profile with peaks at $0 \mathrm{~km} \mathrm{~s}^{-1}$ and $\sim 350 \mathrm{~km} \mathrm{~s}^{-1}$ (see top right in Fig. 3). Moment-0 maps of the channels containing the emission of the first (see bottom left in Fig. 3) and second (see bottom middle in Fig. 3) peaks reveal a spatial mis-alignment of the two [CI]2-1 peaks, which suggests two components. The emission that corresponds to the $0 \mathrm{~km} \mathrm{~s}^{-1}$ peak is at the location of the $\mathrm{HzRG}$ (component 1, indicated with a blue cross in Fig. 3), while the $\sim 350 \mathrm{~km} \mathrm{~s}^{-1}$ gas is shifted 0.5 to the south-east (component 2, indicated with the red cross in Fig. 3). A broad underlying component is visible in the spectrum for component 2. Unfortunately, the spatial resolution of the data and the broad underlying component do not allow for a clearer separation of the two components.

The spectral velocity profile of component 1 , with no overlap with component 2 (see Fig. 3 left), has a Lorentzian-shaped profile, with a full-width at half-maxima (FWHM) of $270 \pm$ $15 \mathrm{~km} \mathrm{~s}^{-1}$ and a redshift of $z=2.1606 \pm 0.0041$, which agrees 

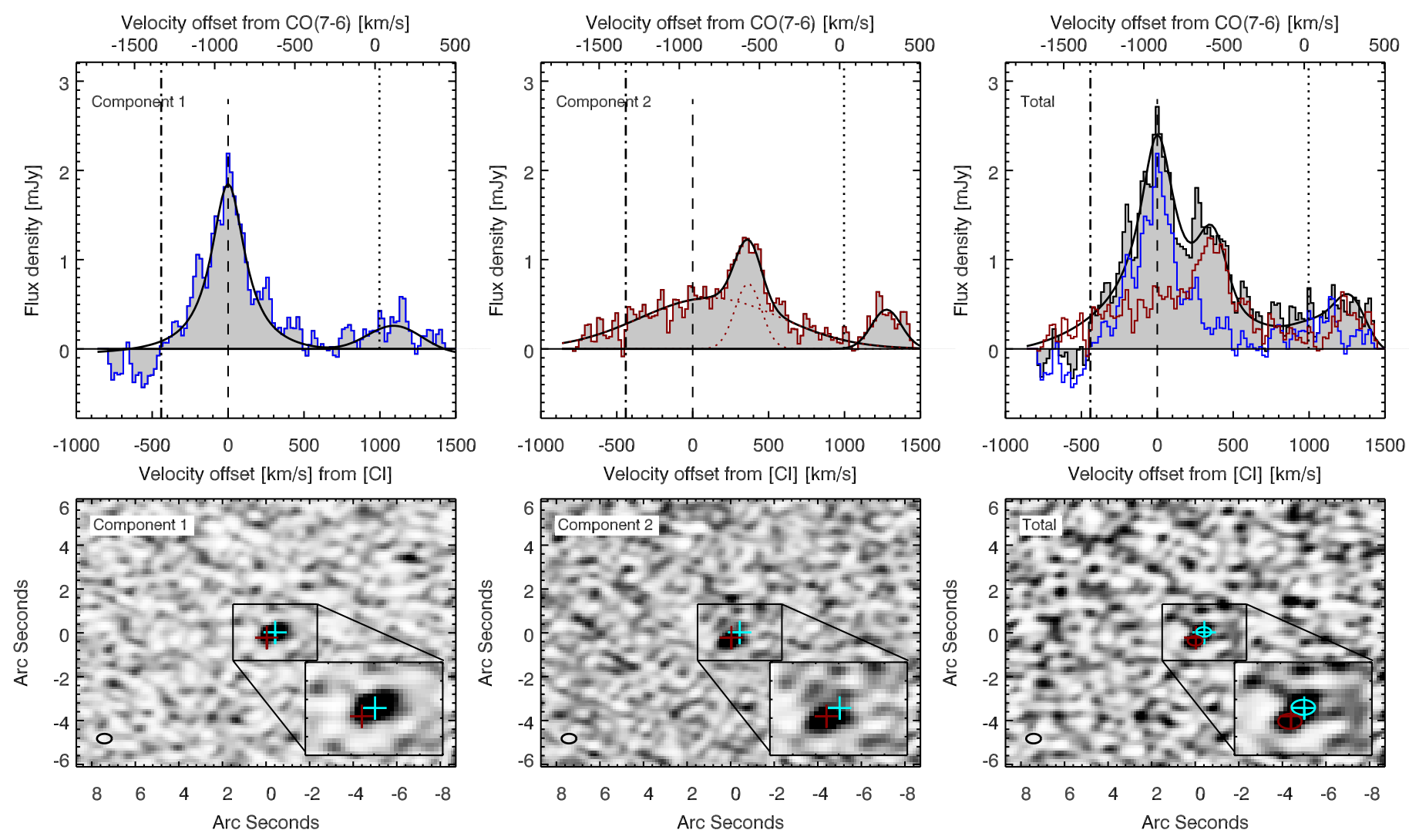

Fig. 3. $[\mathrm{CI}] 2-1$ spectra and moment- 0 maps for component 1 , component 2 , and the total $[\mathrm{CI}] 2-1$ and $\mathrm{CO}(7-6)$ emission. Top row: the spectra extracted from beam-sized areas for component 1 , component 2 , and the total. The areas from which the spectra of component 1 and component 2 are extracted do not overlap. The Lorentzian profile for the component 1 [CI]2-1 line (top left), the double Gaussian profile for the component 2 [CI]2-1 line (top middle), and the single Gaussian fits for the two $\mathrm{CO}(7-6)$ lines are over-plotted as black curves. The sum of the Lorentzian and Gaussian profiles is over-plotted in black in the total spectrum (top right). The dashed lines indicate the 0 -velocity of the [CI]2-1 frequency at $z=2.1606$, and the dotted lines indicate the 0-velocity for the $\mathrm{CO}(7-6)$ frequency at the same redshift. This redshift is in agreement with the redshift determined from the $\mathrm{CO}(1-0)$ line (Emonts et al. 2013). The dotted-dashed line indicate the 0-velocity of the [CI]2-1 frequency at $z=2.156$, determined from the HeII $\lambda 1640 \AA$ line (which, as a non-resonant line, should represent the systemic velocity of the AGN, Humphrey et al. 2008). Bottom row: the moment-0 maps of the [CI]2-1 emission from component 1 (bottom left), component 2 (bottom middle), and the total (bottom right) [CI]2-1 emission and zoom-ins of the centres of the images. The total [CI]2-1 moment- 0 maps is overlaid with [CI]2-1 line contours of component 1 and 2 . The blue and red crosses indicate the peaks of the [CI]2-1 emission of component 1 (blue) and component 2 (red).

with the redshift determined from the $\mathrm{CO}(1-0)$ line (Emonts et al. 2013). We adopt this as the systemic redshift since the [CI]2-1 line has higher spectral resolution and S/N compared to the HeII $11640 \AA$ (Hatch et al. 2008). At the expected frequency of the $\mathrm{CO}(7-6)$ line, we detect a $3.5 \sigma$ Gaussian shaped CO(7-6) emission line with FWHM of $435 \pm 85 \mathrm{~km} \mathrm{~s}^{-1}$. The broadness of the $[\mathrm{CI}] 2-1$ line makes the spectroscopic separation of the $[\mathrm{CI}] 2-1$ and $\mathrm{CO}(7-6)$ lines difficult, as very few linefree channels separate them. To avoid the lines contaminating each other, the velocity integrated line flux for the $[\mathrm{CI}] 2-1$ line is calculated by integrating the fitted profile from $-610 \mathrm{~km} \mathrm{~s}^{-1}$ to $530 \mathrm{~km} \mathrm{~s}^{-1}$, while the integrated $\operatorname{CO}(7-6)$ line flux is integrated from $690 \mathrm{~km} \mathrm{~s}^{-1}$ to $1390 \mathrm{~km} \mathrm{~s}^{-1}$. The $\mathrm{CO}(7-6)$ to $[\mathrm{CI}] 2-1$ line luminosity ratio is 0.2 . The peak ratio of the two [CI]2-1 components is $\sim 3$ times lower than the ratio of the $246 \mathrm{GHz}$ continuum at these positions, implying that component 2 is relatively brighter in [CI] $2-1$ than in $246 \mathrm{GHz}$ emission.

The velocity profile of component 2 shows evidence for two components, one broad and one much narrower component (see Fig. 3). The best two-component Gaussian fit has a FWHM of $1100 \pm 65 \mathrm{~km} \mathrm{~s}^{-1}$ for the broad, and $230 \pm 35 \mathrm{~km} \mathrm{~s}^{-1}$ for the narrow components. The centre of the narrow component is shifted $\sim 360 \mathrm{~km} \mathrm{~s}^{-1}$ redward of the systemic velocity of the $[\mathrm{CI}] 2-1$ line for component 1 . The $\mathrm{CO}(7-6)$ emission line is also detected for component 2 , at a level of $4.8 \sigma$, which is more significant than the $\mathrm{CO}(7-6)$ detection for component 1 . The $\mathrm{CO}(7-6)$ line peaks at $\sim 1275 \mathrm{~km} \mathrm{~s}^{-1}$ relative to the centre of the $[\mathrm{CI}] 2-1$ line, corresponding to a rest velocity for the $\mathrm{CO}(7-6)$ line of $\sim 280 \mathrm{~km} \mathrm{~s}^{-1}$. The narrow component of the $[\mathrm{CI}] 2-1$ line and the $\mathrm{CO}(7-6)$ line both have an offset of $80 \mathrm{~km} \mathrm{~s}^{-1}$ relative to the systemic redshift of component 1 . Just as for component 1 , separating the two lines spectroscopically is difficult and we therefore calculate the integrated flux of the [CI]2-1 line from $-825 \mathrm{~km} \mathrm{~s}^{-1}$ to $990 \mathrm{~km} \mathrm{~s}^{-1}$ and the integrated flux of the $\mathrm{CO}(7-6)$ line from $1000 \mathrm{~km} \mathrm{~s}^{-1}$ to $1490 \mathrm{~km} \mathrm{~s}^{-1}$. We additionally derive the flux of the narrow and broad components separately, by integrating the fitted Gaussian profiles. The $\mathrm{CO}(7-6)$ to $[\mathrm{CI}] 2-1$ line luminosity ratio is 0.14 , lower than for component 1 . The two components have a $[\mathrm{CI}] 2-1$ line peak ratio of 1.7 , and a $\mathrm{CO}(7-6)$ line peak ratio of 0.4 .

The total spectrum of the two components (see Fig. 3 right) clearly shows a double peaked $[\mathrm{CI}] 2-1$ line and a broad CO(7-6) line. We sum the Lorentzian fit of component 1 and the two Gaussian fits of component 2, which results in a profile that fits the observed integrated $[\mathrm{CI}] 2-1$ line of both components well. We likewise sum the two single Gaussians fitted to the $\operatorname{CO}(7-6)$ lines in component 1 and component 2 . The full 


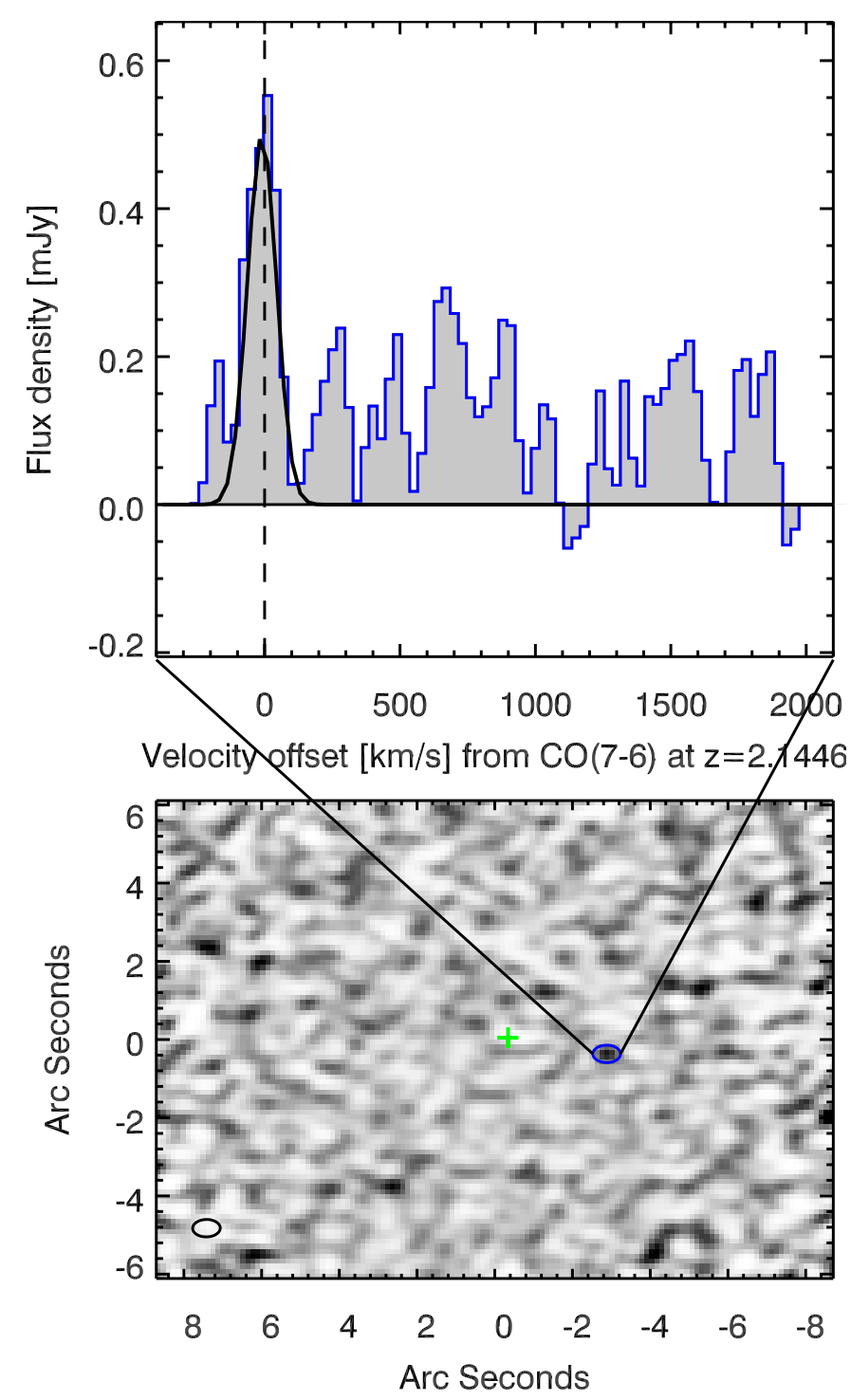

Fig. 4. Bottom panel: moment-0 map of the $\mathrm{CO}(7-6)$ emission at the position of the brightest companion. Top panel: spectrum extracted from the beam-size area shown by the blue ellipse at the position of the companion sources in the bottom panel and is binned to $30 \mathrm{~km} \mathrm{~s}^{-1}$ channels The spectrum shows both the continuum and $\mathrm{CO}(7-6)$ line emission. The redshift of the line is consistent with the optical $z=2.1446$ (Kuiper et al. 2011), which was taken as zero velocity in the spectrum.

[CI]2-1 plus $\mathrm{CO}(7-6)$ profile is over-plotted on the full spectrum in the top right panel of Fig. 3. The $\mathrm{CO}(7-6)$ line in the total spectrum is even broader than for component 1 and 2, making the separation even more difficult. We therefore calculate the [CI]2-1 integrated line flux from $-590 \mathrm{~km} \mathrm{~s}^{-1}$ to $700 \mathrm{~km} \mathrm{~s}^{-1}$ and the $\mathrm{CO}(7-6)$ integrated line flux is integrated from $700 \mathrm{~km} \mathrm{~s}^{-1}$ to $1390 \mathrm{~km} \mathrm{~s}^{-1}$. The $\mathrm{CO}(7-6)$ to $[\mathrm{CI}] 2-1$ line luminosity ratio for the total spectrum is 0.2 , the same as for component 1 .

\subsubsection{The companion source}

We detect line emission in companion \#10 20 kpc to the west of the $\mathrm{H}_{z} \mathrm{RG}$ (see Fig. 4). SINFONI spectroscopy of this companion shows line emission from $\mathrm{H} \alpha$, [OII] and [OIII], and that companion \#10 has a velocity offset of $\sim-1360 \mathrm{~km} \mathrm{~s}^{-1}$, which corresponds to a redshift of $z=2.1446$ (Kuiper et al. 2011). Unfortunately, the $[\mathrm{CI}] 2-1$ is shifted out of the observed band at this redshift and the $\mathrm{H}_{2} \mathrm{O}$ line falls in a gap in the response of the band. However, the $\mathrm{CO}(7-6)$ line is offset by $997 \mathrm{~km} \mathrm{~s}^{-1}$ from the $[\mathrm{CI}] 2-1$ line and therefore still lies within the band at this redshift. We identify the detected emission line as $\mathrm{CO}(7-6)$ line emission from companion \#10 (see Fig. 4). It is therefore highly likely that the $246 \mathrm{GHz}$ continuum emission that we detect at this position is related to companion \#10. This $\operatorname{CO}(7-6)$ line can be fitted with a Gaussian profile with a FWHM of $130 \pm 20 \mathrm{~km} \mathrm{~s}^{-1}$ and the integrated line flux from $-170 \mathrm{~km} \mathrm{~s}^{-1}$ to $150 \mathrm{~km} \mathrm{~s}^{-1}$ of $0.08 \pm 0.01 \mathrm{Jy} \mathrm{km} \mathrm{s}^{-1}$ (Table 2). We do not detect any line emission at the position of the tentative $246 \mathrm{GHz}$ continuum companion \#8.

\section{3. $\mathrm{H}_{2} \mathrm{O}$ line emission}

We detect emission from the $\mathrm{H}_{2} \mathrm{O} 2_{11}-2_{02}$ transition $\sim 25 \mathrm{kpc}$ to the west and $\sim 50 \mathrm{kpc}$ to the east of the radio core at the expected observed frequency for $\mathrm{H}_{2} \mathrm{O}$ at $z=2.1606$. The western $4 \sigma$ detection is located just south of the strongest $246 \mathrm{GHz}$ continuum companion, at the bend of the radio jet, i.e. at radio knot B4 (see Fig. 5). The eastern 3.7 $\sigma$ detection is located west of the radio knot $\mathrm{A}$ (see Fig. 5). To establish that this emission is real, we perform a Jackknife test, cutting the observed time in half. The two $\mathrm{H}_{2} \mathrm{O}$ lines show up in both halves of the data with $\mathrm{a} \gtrsim 2 \sigma$ significance, which suggests that the emission lines are real and not simply noise peaks. Fitting Gaussian profiles to the emission lines result in FWHMs and relative velocities of $230 \pm 50$ and $160 \pm 20 \mathrm{~km} \mathrm{~s}^{-1}$ for the western detection and $350 \pm 70$ and $125 \pm 30 \mathrm{~km} \mathrm{~s}^{-1}$ for the eastern detection respectively. No $246 \mathrm{GHz}$ continuum emission is detected at the positions of the two $\mathrm{H}_{2} \mathrm{O}$ detections down to an rms of $40 \mu \mathrm{Jy}$, and no $\mathrm{H}_{2} \mathrm{O}$ emission line is detected at the position of the $\mathrm{H}_{z} \mathrm{RG}$ (see Fig. 5) down to an rms of $40 \mu \mathrm{Jy}$ in $60 \mathrm{~km} \mathrm{~s}^{-1}$ wide channels; assuming that the $720 \mathrm{~km} \mathrm{~s}^{-1}$ width of the $\operatorname{CO}(7-6)$ line from the total spectrum yields a $3 \sigma$ upper limit of $0.9 \mathrm{Jy} \mathrm{km} \mathrm{s}^{-1}$.

\section{Analysis and discussion}

The lines we detected in the Spiderweb galaxy are useful for a wide range of gas diagnostics. The atomic forbidden line of carbon, [CI]2-1, is a good tracer of relatively diffuse, low extinction molecular gas (e.g. Papadopoulos et al. 2004), since its line strength is linearly proportional to the column density of molecular gas (Glover et al. 2015). $\mathrm{CO}(7-6)$ emission is strong in dense, highly excited optically thick molecular gas. The thermal dust continuum over the range of a few $100 \mathrm{GHz}$ represents the cooling of dust that is heated by the intense stellar and AGN radiation fields within the Spiderweb galaxy. The $\mathrm{H}_{2} \mathrm{O} 2_{11}-2_{02}$ line is excited either in slow shocks $\left(10-40 \mathrm{~km} \mathrm{~s}^{-1}\right)$ in dense molecular gas $\left(10^{3-5} \mathrm{~cm}^{-3}\right.$; Flower \& Pineau Des Forêts 2010) or by IR pumping (e.g. van der Werf et al. 2011). Thus our data, in principle, probe a wide range of conditions and heating/excitation mechanisms in (relatively dense) molecular and atomic gas.

\subsection{Diffuse and dense molecular gas}

\subsubsection{Line velocity profiles}

Fitting of Lorentzian and Gaussian profiles for the emission line shows that the $[\mathrm{CI}] 2-1$ and $\mathrm{CO}(7-6)$ lines component 1 and 2 have very different velocity profiles. Figure 6 compares the velocity profiles of the $[\mathrm{CI}] 2-1$ and $\mathrm{CO}(7-6)$ emission lines for component 1 (left), component 2 (middle), and the total (right). Both the $[\mathrm{CI}] 2-1$ and $\mathrm{CO}(7-6)$ emission lines of component 1 
B. Gullberg et al.: Dew drops in a dusty web

Table 2. $\mathrm{H}_{2} \mathrm{O}$, $[\mathrm{CI}] 2-1$ and $\mathrm{CO}(7-6)$ emission line positions, velocity integrated fluxes, and FWHMs.

\begin{tabular}{|c|c|c|c|c|c|c|}
\hline \multirow{2}{*}{ Transition } & \multirow{2}{*}{$\begin{array}{c}\text { Frequency } \\
\text { GHz }\end{array}$} & \multicolumn{2}{|c|}{ Position } & \multirow{2}{*}{$\begin{array}{c}S \mathrm{~d} V \\
\mathrm{Jy} \mathrm{km} \mathrm{s}^{-1}\end{array}$} & \multicolumn{2}{|c|}{$F W H M$} \\
\hline & & RA & Dec & & $\mathrm{km} \mathrm{s}^{-1}$ & $\mathrm{~km} \mathrm{~s}^{-1}$ \\
\hline \multicolumn{7}{|l|}{ West } \\
\hline $\mathrm{H}_{2} \mathrm{O}$ & 752.03 & $11: 40: 48.82$ & $-26: 29: 9.580$ & $0.05 \pm 0.01$ & $230 \pm 50$ & - \\
\hline \multicolumn{7}{|l|}{ East } \\
\hline $\mathrm{H}_{2} \mathrm{O}$ & 752.03 & $11: 40: 48.11$ & $-26: 29: 9.970$ & $0.1 \pm 0.02$ & $350 \pm 70$ & - \\
\hline \multicolumn{7}{|c|}{ Component I } \\
\hline $\mathrm{CO}(7-6)$ & 806.65 & $11: 40: 48.33$ & $-26: 29: 8.582$ & $0.13 \pm 0.02$ & $435 \pm 85$ & - \\
\hline$[\mathrm{CI}] 2-1$ & 809.34 & $11: 40: 48.33$ & $-26: 29: 8.582$ & $0.66 \pm 0.02$ & $270 \pm 15$ & - \\
\hline \multicolumn{7}{|c|}{ Component 2} \\
\hline $\mathrm{CO}(7-6)$ & 806.65 & $11: 40: 48.36$ & $-26: 29: 8.822$ & $0.11 \pm 0.01$ & $230 \pm 35$ & - \\
\hline$[\mathrm{CI}] 2-1$ & 809.34 & $11: 40: 48.36$ & $-26: 29: 8.822$ & $0.79 \pm 0.03^{a}$ & $1100 \pm 65$ & $205 \pm 20$ \\
\hline \multicolumn{7}{|c|}{ Component I + component 2} \\
\hline $\mathrm{H}_{2} \mathrm{O}$ & 752.03 & $11: 40: 48.33$ & $-26: 29: 8.582$ & $<0.9^{b}$ & - & - \\
\hline $\mathrm{CO}(7-6)$ & 806.65 & $11: 40: 48.33$ & $-26: 29: 8.582$ & $0.28 \pm 0.02$ & $720 \pm 110$ & - \\
\hline$[\mathrm{CI}] 2-1$ & 809.34 & $11: 40: 48.33$ & $-26: 29: 8.582$ & $1.30 \pm 0.03$ & $420 \pm 30$ & $280 \pm 40$ \\
\hline \multicolumn{7}{|c|}{ Companion \#10 } \\
\hline $\mathrm{CO}(7-6)$ & 806.65 & $11: 40: 48.15$ & $-26: 29: 09.075$ & $0.08 \pm 0.01$ & $130 \pm 20$ & - \\
\hline
\end{tabular}

Notes. Fluxes and fitted FWHMs are given for $[\mathrm{CI}] 2-1$ component 1,2 , total, and the $\mathrm{H}_{2} \mathrm{O}$ components. The spectra are extracted within a synthesised beam size which, for the [CI] $2-1$ observations, is $0^{\prime \prime} .72 \times 00^{\prime \prime} .45$ with pa $87.4^{\circ}$, and for the $\mathrm{H}_{2} \mathrm{O}$ observations is $0^{\prime \prime}$.94 $\times 0$ 0'56 with $\mathrm{pa}-84.1^{\circ}{ }^{(a)}$ The $[\mathrm{CI}] 2-1$ flux is composed of $0.63 \pm 0.03 \mathrm{Jy} \mathrm{km} \mathrm{s}^{-1}$ for the broad velocity gas, and $0.16 \pm 0.03 \mathrm{Jy} \mathrm{km} \mathrm{s}^{-1}$ for the narrow velocity gas. ${ }^{(b)}$ The $3 \sigma$ upper limit of the $\mathrm{H}_{2} \mathrm{O}$ emission taking is taken to be $3 \times$ the rms in $60 \mathrm{~km} \mathrm{~s}^{-1}$ wide channels and assuming a width of the line as being that of the $\mathrm{CO}(7-6)$ line.

are broad, but though $[\mathrm{CI}] 2-1$ and $\mathrm{CO}(7-6)$ do not trace exactly the same phases of the molecular gas, the broadness of the two lines does suggest that the two phases are related. The velocity profiles of the $[\mathrm{CI}] 2-1$ and $\mathrm{CO}(7-6)$ lines for component 2 show similarities between the narrow [CI]2-1 component and the $\mathrm{CO}(7-6)$ line. The broad $[\mathrm{CI}] 2-1$ component is not seen in the $\mathrm{CO}(7-6)$ line because of both the limited spectral coverage of our observations on the high velocity end of our bandpass, and limited $\mathrm{S} / \mathrm{N}$ of this faint line. The fact that the narrow velocity gas in component 2 is detected in both [CI]2-1 and $\mathrm{CO}(7-6)$ emission and with similar FWHM indicates that this is a well-defined object. High spatial resolution observations would be required to determine if this is a separate self-gravitating object, or a kinematic feature within the same physical object. The broadness of the $\operatorname{CO}(7-6)$ emission in the total spectrum indicates that this emission is dominated by the $\mathrm{CO}(7-6)$ emission from component 1 .

The $\mathrm{CO}(7-6)$ lines for both component 1 and 2 , show an offset from the systemic redshift of $\sim 90-100 \mathrm{~km} \mathrm{~s}^{-1}$. A similar offset between the [CI] and $\mathrm{CO}$ lines is seen in a study of H1413+117 (the Cloverleaf Galaxy) by Weiß et al. (2003). They conclude that the reason for the shift is unclear, but that gravitational amplification should not alter the frequency of the lines, unless the distribution of the $[\mathrm{CI}]$ and $\mathrm{CO}$ emitting gas is different. Because the $[\mathrm{CI}]$ and $\mathrm{CO}$ lines for the Cloverleaf Galaxy have low S/N, Weiß et al. (2003) conclude that high S/N observations are necessary to confirm the offset and its origin, but that, if it is genuine, it is most likely due to opacity effects. Since we see a similar offset for the Spiderweb galaxy, which is an unlensed source, differential lensing cannot be the cause of this offset, however, differences in the opacity of the two lines $f$. The $\mathrm{CO}(7-6)$ line has a higher optical depth than the $[\mathrm{CI}] 2-1$ line $\left(\tau_{[\mathrm{CI}]}=0.1\right.$, Weiß et al. 2003), meaning that the $\mathrm{CO}(7-6)$ emitted photons go through internal absorption and emission. The line photons, whose frequencies have been shifted away from the systemic frequency of the $\operatorname{CO}(7-6)$ emission line, have a higher probability of escaping, thus shifting the overall observed frequency.

The $\mathrm{H} \alpha$ line for the Spiderweb galaxy was detected with SINFONI (Nesvadba et al. 2006) and ISAAC (Humphrey et al. 2008). The H $\alpha$ ( $\lambda$ 6550), [NII] ( $\lambda$ 6585) lines, and [SII] ( $\lambda \lambda 6718$, 6733 ) doublet are spectrally very close and the lines are therefore blended together. Both studies fit the blended lines with multiple Gaussians. Nesvadba et al. (2006) finds $F W H M=14900 \mathrm{~km} \mathrm{~s}^{-1}$ for the AGN component and $F W H M<2400 \mathrm{~km} \mathrm{~s}^{-1}$ for the emission originating from a region that surrounds the AGN. This is the same order of magnitude that Humphrey et al. (2008) find by fitting six Gaussian profiles, including two $\mathrm{H} \alpha$ components. These two $\mathrm{H} \alpha$ components consist of a transmitted broad line region from the AGN $\left(F W H M=13900 \pm 500 \mathrm{~km} \mathrm{~s}^{-1}\right)$, and a component with $F W H M=1200 \pm 80 \mathrm{~km} \mathrm{~s}^{-1}$. The relatively low spectral resolution of the ISAAC spectrum does not allow for a further de-blending of the lines. However, the $\mathrm{H} \alpha$ line profile does show the presence of a broad and narrow component, similar to what we observe in the $[\mathrm{CI}] 2-1$ emission of component 2 . This strengthens the idea that the $[\mathrm{CI}] 2-1$ emission traces the bulk of the gas in the galaxy, which is consistent with the [CI] emitting gas being distributed throughout and, generally, tracing gas of moderate extinctions and relatively low density molecular gas (Papadopoulos et al. 2004).

\subsubsection{Line ratios}

The utility of using the [CI] emission lines as tracers of the $\mathrm{H}_{2}$ column density has been examined by Papadopoulos et al. (2004) by considering the [CI $2-1$ to $\mathrm{CO}$ line ratio. The $[\mathrm{CI}]$ lines, $[\mathrm{CI}]{ }^{3} \mathrm{P}_{1} \rightarrow{ }^{3} \mathrm{P}_{0}([\mathrm{CI}] 1-0)$ and $[\mathrm{CI}]{ }^{3} \mathrm{P}_{2} \rightarrow{ }^{3} \mathrm{P}_{1}([\mathrm{CI}] 2-1)$, have critical densities of $n_{\mathrm{cr},[\mathrm{Cl}] 1-0} \sim 500 \mathrm{~cm}^{-3}$ and $n_{\mathrm{cr},[\mathrm{CI}] 2-1} \sim$ $10^{3} \mathrm{~cm}^{-3}$, similar to that of $\mathrm{CO}(1-0)$ and $\mathrm{CO}(2-1)$, which are often used as $\mathrm{H}_{2}$ tracers. The [CI] lines have low to moderate optical depths $(\tau \approx 0.1-1)$, which means that these lines have the advantage, compared to $\mathrm{CO}$, of tracing higher column-density 


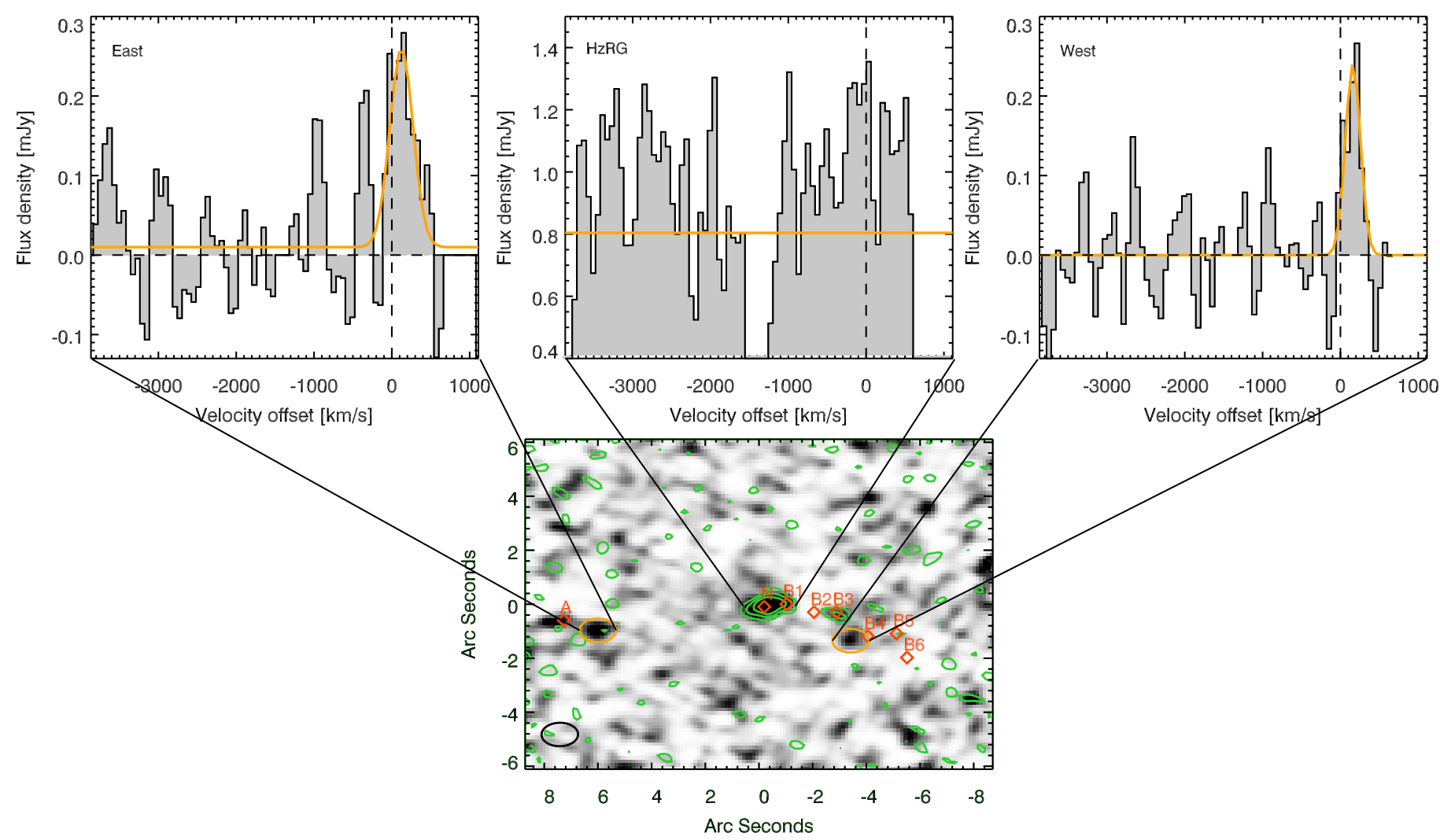

Fig. 5. Non-continuum subtracted $\mathrm{H}_{2} \mathrm{O}$ spectra for the detection of the west and east and the non-detection at the position at the $\mathrm{Hz}$ RG and $\mathrm{H}_{2} \mathrm{O}$ moment-0 map. Top left panel: $\mathrm{H}_{2} \mathrm{O}$ line detected $\sim 50 \mathrm{kpc}$ to the east of the radio core, has a $3.7 \sigma$ significance. The emission is located due west of the knot $\mathrm{A}$ in the radio jet. The fitted Gaussian is over-plotted in orange. Top middle panel: spectrum at the position of the radio core, shows no detection of $\mathrm{H}_{2} \mathrm{O}$ emission - only continuum emission. A small separation between the two spectral windows results in the gap in the continuum between -1300 and $-1500 \mathrm{~km} \mathrm{~s}^{-1}$. Top right panel: $\mathrm{H}_{2} \mathrm{O}$ line detected $\sim 25 \mathrm{kpc}$ to the west of the radio core, showing a $4 \sigma$ $\mathrm{H}_{2} \mathrm{O}$ detection at the expected frequency for $z=2.161$. The emission is located at the bend of the radio jet, B4 (Pentericci et al. 1997). The best-fit Gaussian is over-plotted in orange. Bottom panel: moment- 0 map of the $\mathrm{H}_{2} \mathrm{O}$ emission (without continuum subtraction), overlaid with the $246 \mathrm{GHz}$ continuum emission in green contours. The orange ellipses show the $\mathrm{H}_{2} \mathrm{O}$ emission and the orange-red diamonds the positions of the knots in the radio jet, given by Pentericci et al. (1997).

cold diffuse molecular gas. The $[\mathrm{CI}]$ lines are also more easily excited. These properties make the $[\mathrm{CI}]$ lines more direct tracers of the molecular gas mass than the optically thick ${ }^{12} \mathrm{CO}$ lines. Though the $\mathrm{H}_{2}$-tracing capability of $[\mathrm{CI}]$ decreases for low metallicities, Papadopoulos et al. (2004) conclude it is still a better $\mathrm{H}_{2}$ tracer than ${ }^{12} \mathrm{CO}$ (see also Glover et al. 2015).

The ground-state transition, [CI]1-0, is the most direct $\mathrm{H}_{2}$ gas-mass tracer of the two [CI] lines because it is (relatively) less sensitive to the excitation conditions of the gas. However, the [CI]2-1 is still a much better tracer of the $\mathrm{H}_{2}$ gas than the $\mathrm{CO}(7-6)$ line at a similar frequency. The much higher critical density of the $\mathrm{CO}(7-6)$ line of $n_{\mathrm{cr}, \mathrm{CO}(7-6)} \sim 3 \times 10^{6} \mathrm{~cm}^{-3}$ and higher excitation energy of $E_{76} / k \sim 155 \mathrm{~K}$, compared to $E_{21} / k \sim 62 \mathrm{~K}$ for the $[\mathrm{CI}] 2-1$ line, means that the [CI]2-1 and $\mathrm{CO}(7-6)$ lines are unlikely to trace exactly the same molecular gas phases. The $\mathrm{CO}(7-6)$ line is likely tracing the higher density, more highly excited molecular gas.

We searched the literature for high- $z$ sources with observations of both the [CI]2-1 and $\mathrm{CO}(7-6)$ emission lines and determined IR luminosities, and find nine lensed sub-millimetre galaxies (SMG) and QSOs (see Table 3). To compare the lensed sources and the un-lensed Spiderweb galaxy, which have different luminosities, we normalise the $[\mathrm{CI}] 2-1$ and $\mathrm{CO}(7-6)$ line luminosities by the IR luminosity, which cancels out the lensing magnification for the lensed sources (assuming there is no differential lensing between the IR and line emission). Figure 7 shows a positive correlation between these normalised line luminosities. The only outlier in the comparison sample is the highly lensed Cloverleaf Galaxy (Alloin et al. 1997; Weiß et al. 2003; van der Werf et al. 2011). The Spiderweb galaxy is an outlier, but on the low side of the correlation.

\subsubsection{Possible causes of observed line ratio}

The Spiderweb galaxy is, like the Cloverleaf galaxy, an outlier, compared to lensed galaxies. This unusually low $\mathrm{CO}(7-6) /[\mathrm{CI}] 2-1$ line ratio could be due to the Spiderweb galaxy being an extraordinary $\mathrm{H} z \mathrm{RG}$ with a highly unusual ratio between the diffuse and dense molecular gas, or due to the impact of cosmic-ray heating and ionisation, or differential lensing.

$$
\text { Comparing } L_{\mathrm{CO}(7-6)}^{\prime}=1.2 \times 10^{10} \mathrm{~K} \mathrm{~km} \mathrm{~s}^{-1} \mathrm{pc}^{2} \text { with }
$$
$L_{\mathrm{CO}(1-0)}^{\prime}=6.5 \times 10^{10} \mathrm{~K} \mathrm{~km} \mathrm{~s}^{-1} \mathrm{pc}^{2}$ (Emonts et al. 2013), we find that the $\operatorname{CO}(7-6)$ emission is only $18 \%$ of the value for thermalised gas. This indicates that the molecular gas phase is not thermalised and dominated by the cold and diffuse gas, which is traced by the $[\mathrm{CI}] 2-1$ emission line ${ }^{1}$. Alternatively, a fraction of the $\mathrm{CO}$ in the Spiderweb galaxy may be dissociated

1 Note, however, that the high spatial resolution of the ALMA observation means that up to $2 / 3$ of the $\mathrm{CO}(7-6)$ emission may not be detected owing to lack of sensitivity to very extended, low surface-brightness line emission (Emonts et al., in prep.). 

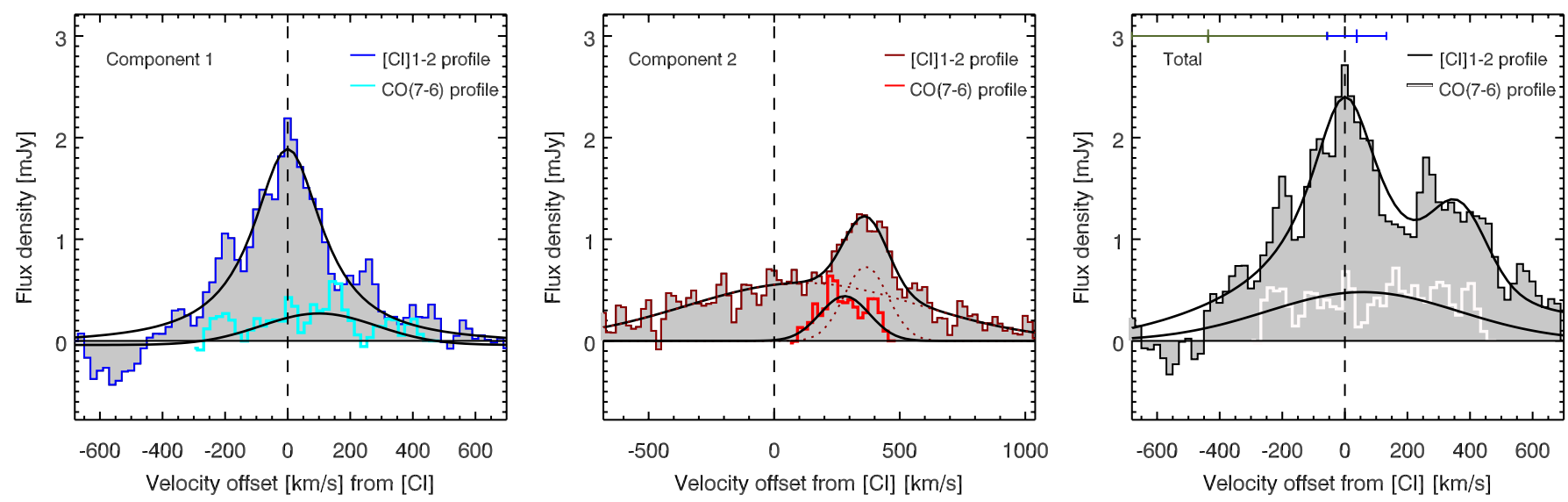

Fig. 6. $[\mathrm{CI}] 2-1$ spectra (dark blue, dark red and black histograms) for component 1, component 2, and the total over-plotted with the respective $\mathrm{CO}(7-6)$ lines (light blue, orange and white histograms) to compare the velocity profiles. The fitted profiles from Sect. 3 are over-plotted as black curves, with the individual velocity components as dotted lines. The narrow [CI]2-1 component of component 2 is offset by $365 \mathrm{~km} \mathrm{~s}^{-1}$ from the systemic redshift. The bar above the velocity profiles in the right plot, marked the HeII redshift and errors (olive bar) from Röttgering et al. (1997) and the $\mathrm{CO}(1-0)$ redshift and error (blue bar) from Emonts et al. (2013).

Table 3. SMGs and QSOs from the literature with published [CI]2-1, $\mathrm{CO}(7-6)$ and $\mathrm{H}_{2} \mathrm{O}$ detections along with published IR luminosities used for comparison to the Spiderweb galaxy.

\begin{tabular}{lccccl}
\hline \hline Source & $z$ & $\mu$ & $\begin{array}{c}S \mathrm{~d} V_{\mathrm{CO}(7-6)} \\
{\left[\mathrm{Jy} \mathrm{km} \mathrm{s}^{-1}\right]}\end{array}$ & $\begin{array}{c}S \mathrm{~d} V_{[\mathrm{CC}]} \\
{\left[\mathrm{Jy} \mathrm{km} \mathrm{s}^{-1}\right]}\end{array}$ & Reference \\
\hline SDP.11 & 1.7860 & 18 & $18 \pm 14$ & $31 \pm 14$ & Lupu et al. (2012), Ferkinhoff et al. (2014) \\
SDP17.b & 2.3080 & 4.3 & $11 \pm 7$ & $13 \pm 7$ & Lupu et al. (2012) \\
SMMJ2135-0102 & 2.3259 & 32.5 & $12.6 \pm 0.6$ & $16.2 \pm 0.6$ & Danielson et al. (2011) \\
SMMJ16359+6612 & 2.5160 & 22 & $3.3 \pm 1.4$ & $1.6 \pm 0.3$ & Kneib et al. (2005), Walter et al. (2011) \\
H1413+117 & 2.5585 & 11 & $44.6 \pm 3.1$ & $5.2 \pm 0.3$ & Alloin et al. (1997), Weiß et al. (2003) \\
SMMJ14011+0252 & 2.5650 & 23 & $3.2 \pm 0.5$ & $3.1 \pm 0.3$ & Downes \& Solomon (2003), Walter et al. (2011) \\
SDP.81 & 3.0370 & 9.5 & $12 \pm 4$ & $<6.5$ & Lupu et al. (2012) \\
MM18423+5938 & 3.9296 & 12 & $3.9 \pm 0.5$ & $4.2 \pm 0.8$ & Lestrade et al. (2010) \\
ID141 & 4.2430 & $10-30$ & $6.5 \pm 1.4$ & $3.4 \pm 1.1$ & Cox et al. (2011) \\
HFLS3 & 6.3369 & 2.2 & $2.2 \pm 0.3$ & $0.5 \pm 0.4$ & Riechers et al. (2013) \\
\hline & & & $S \mathrm{~d} V_{\mathrm{H}_{2} \mathrm{O}}$ & $\mu L_{\mathrm{IR}}$ & Reference \\
& & & {$\left[\mathrm{Jy} \mathrm{km} \mathrm{s} \mathrm{k}^{-1}\right]$} & {$\left[10^{13} L_{\odot}\right]$} & \\
\hline SDP9 & 1.574 & 8.5 & $14.4 \pm 1.1$ & 4.4 & Lupu et al. (2012) \\
NAv1144 & 2.202 & 5.3 & $7.5 \pm 0.9$ & 5.7 & Omont et al. (2013) \\
G15v2779 & 4.244 & 4.1 & $4.1 \pm 0.6$ & 8.5 & Omont et al. (2013) \\
APM08279+5255 & 3.912 & 4.0 & $<1.1$ & 20 & van der Werf et al. (2011), Walter et al. (2011) \\
HFLS3 & 6.3369 & 2.2 & $2.6 \pm 0.8$ & 4.2 & Riechers et al. (2013) \\
\hline
\end{tabular}

by cosmic-rays, increasing the fraction of atomic carbon in the molecular gas without strongly affecting the excitation of [CI]2-1 or the ionisation state of carbon (Bisbas et al. 2015). In the circum-nuclear region of a powerful radio galaxy, it is likely that cosmic rays may have had a significant impact on the nature of the molecular gas. More observations of other atomic and molecular species in the Spiderweb galaxy are necessary to investigate this quantitatively.

However, since the comparison sample are all lensed galaxies, the difference could also be caused by differential lensing in the comparison sample. Dense (traced by $\mathrm{CO}(7-6)$ ) and diffuse (traced by [CI]2-1) gas in galaxies may be subject to differential lensing, where the emission from the densest gas has a higher overall magnification than the diffuse gas. Serjeant (2012) showed that for lensing magnifications $\mu>2$, the $\mathrm{CO}$ ladder can be strongly distorted by differential lensing. Since all the SMGs and QSOs listed in Table 3 have a magnification $\mu>2$, it may be that their line ratios are all influenced by the affect of differential lensing. If so, this implies that the $\mathrm{CO}(7-6)$ emission probably has a higher magnification than the $[\mathrm{CI}] 2-1$ emission, which would mean that the intrinsic $\mathrm{CO}(7-6) /[\mathrm{CI}] 2-1$ ratio would be lower. We note, however, that although some of the data are limited by $\mathrm{S} / \mathrm{N}$ and/or low resolution, the line profiles of $\mathrm{CO}(7-6)$ and $[\mathrm{CI}] 2-1$ are consistent with each other (see references listed in Table 3). While not conclusive, the similarity of their dynamics suggests that the gas probed by $\mathrm{CO}(7-6)$ and [CI]2-1 follow the same overall dynamics and are perhaps not strongly affected by differential lensing.

We are planning to extend our ALMA [CI] observations to eight $\mathrm{H}_{z} \mathrm{RGs}$ to verify if this low $\mathrm{CO} /[\mathrm{CI}]$ ratio is a common feature and also carry out more detailed studies of the Spiderweb galaxy to investigate this puzzle.

\subsection{Cooling of the post-shock gas owing to slow shocks in molecular halos}

Interestingly, the two regions of $\mathrm{H}_{2} \mathrm{O}$ emission that we detect do not appear to be associated with significant dust continuum 


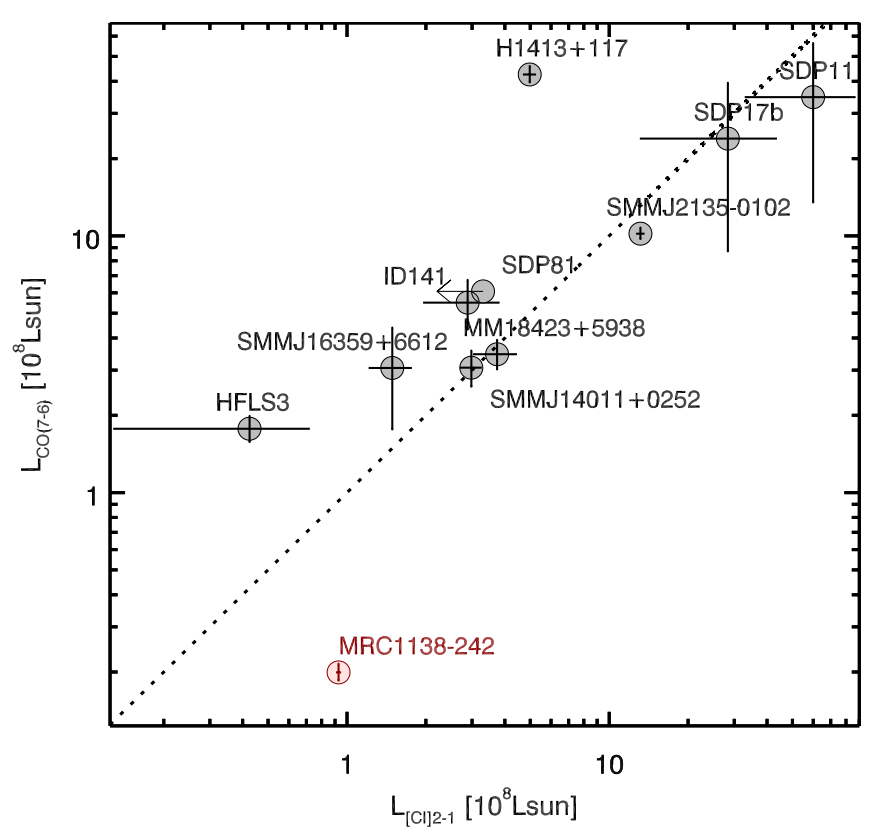

Fig. 7. $\mathrm{CO}(7-6)$ vs. the $[\mathrm{CI}] 2-1$ luminosity for a sample of high- $z$ SMGs and QSO (grey circles) and the Spiderweb galaxy (red circles). The dotted curve is the $1-1$ relation.

emission. This is puzzling since a close association between a dense gas tracer such as $\mathrm{H}_{2} \mathrm{O}$ and dust is expected when the excitation of the $\mathrm{H}_{2} \mathrm{O}$ emission in other high- $z$ (lensed) sources has been attributed to IR pumping (van der Werf et al. 2011; Omont et al. 2013). Moreover, no $\mathrm{H}_{2} \mathrm{O}$ emission is detected at the position of the $\mathrm{H}_{z} \mathrm{RG}$ (Fig. 5), which is surprising, given its strong IR emission and high rate of mechanical energy injection via the radio jets (Fig. 5). Specifically, Omont et al. (2013) detect $\mathrm{H}_{2} \mathrm{O}$ for seven high- $z$ lensed sources from the H-ATLAS survey. Five are detected in $\mathrm{H}_{2} \mathrm{O} 2_{11}-2_{02}$ emission, and three in $\mathrm{H}_{2} \mathrm{O} 2_{02}-1_{11}$ emission. Comparing these $\mathrm{H}_{2} \mathrm{O}$ detections to the $8-1000 \mu \mathrm{m}$ IR luminosity, Omont et al. (2013) find a correlation between the IR and $\mathrm{H}_{2} \mathrm{O}$ line luminosity of $L_{\mathrm{H}_{2} \mathrm{O}}=L_{\mathrm{IR}}^{1.1-1.3}$, with the exact exponent being dependent on which of the two $\mathrm{H}_{2} \mathrm{O}$ lines is used in the analysis. From this relation, they conclude that IR pumping is the most likely mechanism for exciting the water molecules and their line emission. This is consistent with $\mathrm{H}_{2} \mathrm{O}$ tracing dense gas that surrounds regions of intense star formation. Furthermore, a similar conclusion was reached in a more detailed study of the $\mathrm{H}_{2} \mathrm{O}$ emission from the lensed QSO, APM 08279+5255 at $z=3.9$ (van der Werf et al. 2011) and by Yang et al. (2013), who detected $\mathrm{H}_{2} \mathrm{O}$ emission lines in 45 low $z$ galaxies.

Figure 8 plots $L_{\mathrm{H}_{2} \mathrm{O}} / L_{\mathrm{IR}}$ versus $L_{\mathrm{IR}}$ and shows an anticorrelation for IR luminous high- $z$ lensed sources (Omont et al. 2013; van der Werf et al. 2011). SDP.81 is an outlier in this relation, most likely because of an underestimation of the $\mathrm{H}_{2} \mathrm{O}$ luminosity as a result of low $\mathrm{S} / \mathrm{N}$ in the spectrum, and the large extent of the source (Omont et al. 2013). We plot the Spiderweb galaxy as the sum of the $\mathrm{H}_{2} \mathrm{O}$ luminosities of the western and eastern detections and the total infrared luminosity. The $\mathrm{H}_{2} \mathrm{O}$ emission originates from two small regions, and any more widespread emission would not be detected owing to the lack of sensitivity of our ALMA data on larger spatial scales. The IRAM Plateau de Bure observations of the comparison sample of Omont et al. (2013) have two to seven times larger synthesised beam sizes, and may be more sensitive to emission at larger scales. The $L_{\mathrm{H}_{2} \mathrm{O}} / L_{\mathrm{FIR}}$ ratio for the Spiderweb galaxy is therefore a lower

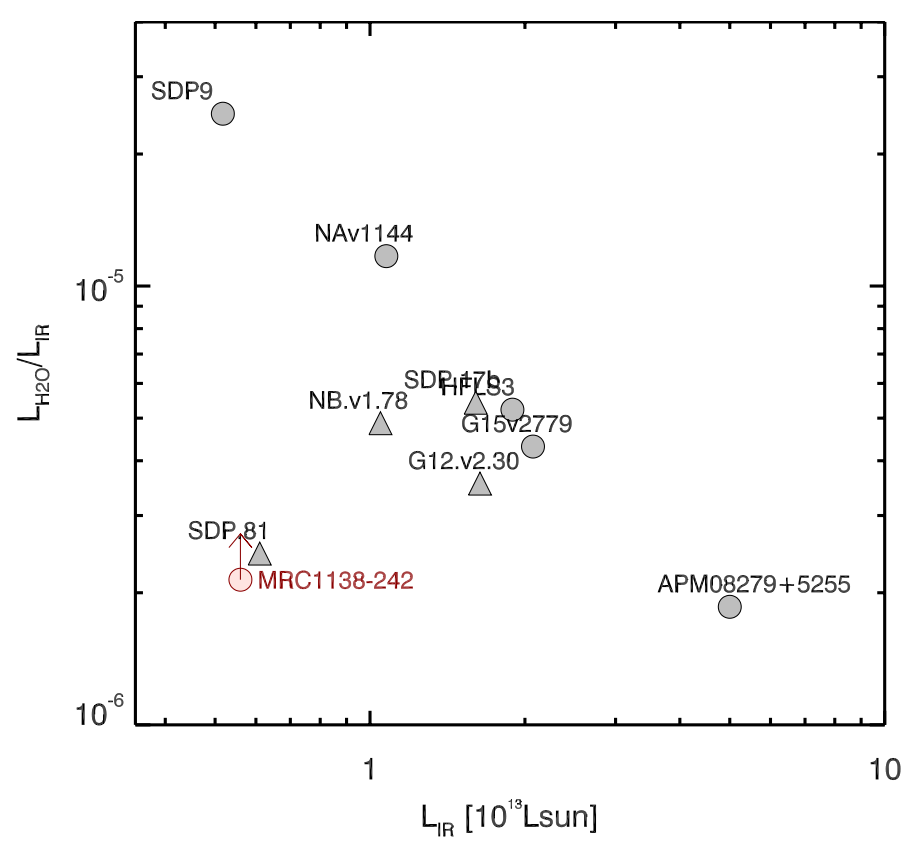

Fig. 8. $L_{\mathrm{H}_{2} \mathrm{O}} / L_{\mathrm{IR}}$-ratio versus intrinsic $L_{\mathrm{IR}}$ for the $\mathrm{H}_{2} \mathrm{O}$ detections from Omont et al. (2013) along with the $\mathrm{H}_{2} \mathrm{O}$ detection for the Spiderweb galaxy. The grey circles are sources from Omont et al. (2013) with directed detections of the $2_{02}-1_{11}$ line and the grey triangles are sources with $2_{11}-2_{02}$ detection, scaled using Mrk231 as a template to get an estimate on the $2_{02}-1_{11}$ luminosity. The Spiderweb galaxy is shown with the red point. We plot the summed $\mathrm{H}_{2} \mathrm{O}$ emission of the western and eastern detection, since the IR luminosity is integrated over the full source.

limit and the Spiderweb galaxy could therefore be an outlier in this trend. The lack of $246 \mathrm{GHz}$ continuum emission at the $\mathrm{H}_{2} \mathrm{O}$ positions, which would imply a much larger deviation from the trend, implies that the emission is not pumped by the IR emission. The $\mathrm{H}_{2} \mathrm{O}$ detection is spatially located at the bend in the radio continuum emission (knot B3) and upstream to the radio jet. Similarly, the eastern $\mathrm{H}_{2} \mathrm{O}$ detection upstream from knot $\mathrm{A}$ in the radio emission is likely associated with the jet, which is projected onto the plane of the sky.

The most straightforward explanation for the $\mathrm{H}_{2} \mathrm{O}$ emission at these positions, the lack of significant associated $246 \mathrm{GHz}$ continuum emission, and the offset from the anti-correlation in Fig. 8, is that the $\mathrm{H}_{2} \mathrm{O}$ emitting gas is shock-heated and represents strong cooling in post-shock heated gas by the passage of the radio jet. $\mathrm{H}_{2} \mathrm{O}$ is one of the main carriers of oxygen along with $\mathrm{CO}$, but has many more radiative degrees of freedom, meaning that it can lose energy efficiently under a wide range of (low energy) excitation conditions and thus contribute significantly to the overall cooling of dense molecular gas, in particular after the shock impact (Flower \& Pineau Des Forêts 2010). Given the small offset of the line peaks from the systemic [CI]2-1 redshift, only 100 to $200 \mathrm{~km} \mathrm{~s}^{-1}$, and the average line width of $290 \mathrm{~km} \mathrm{~s}^{-1}$ of the two $\mathrm{H}_{2} \mathrm{O}$ lines, it is likely that the width of the lines represents the dispersion of the clouds and that mechanical energy is being dissipated within the dense molecular gas. Not only $\mathrm{H}_{2} \mathrm{O}$, but also the detection of strong mid-infrared $\mathrm{H}_{2}$ emission (Ogle et al. 2012), suggest excitation of molecules in lowvelocity shocks (see discussion in Guillard et al. 2009; Appleton et al. 2013). Moreover, we emphasise that, owing to the position of the $\mathrm{H}_{2} \mathrm{O}$ emission in our bandpass, higher negative offset 
velocity emission, such as that from companion \#10, would not lie within our bandpass (Kuiper et al. 2011).

Furthermore, it is unlikely that the dense molecular gas has been entrained during the passage of the radio jet and lifted out of the radio galaxy or any of its companions. There are several reasons why this is unlikely. First, both regions of $\mathrm{H}_{2} \mathrm{O}$ emission are almost at the systemic velocity of the radio galaxy (as determined from $\mathrm{CO}(1-0)$, [CI $] 2-1$ or $\mathrm{CO}(7-6))$. The $\mathrm{H}_{2} \mathrm{O}$ lines have systemic velocities that are significantly lower than any of the companion galaxies with determined redshifts near the sites of $\mathrm{H}_{2} \mathrm{O}$ emission. Second, the cooling time of the dense shock gas is of-order $10^{3}-10^{4} \mathrm{yr}$ for a $20 \mathrm{~km} \mathrm{~s}^{-1}$ shock that was driven into a gas with a density of $10^{5} \mathrm{~cm}^{-3}$ (Flower \& Pineau Des Forêts 2010), which is much shorter than any plausible dynamical time. These facts suggest that the dense molecular gas is forming in situ in the post-shocked gas after the passage of the radio jet. Within this context, it is not surprising to see the emission near the bend in the jet and near the terminal hotspot of the western radio lobe since these are sites where the interaction between the jet and ambient medium are likely to be strong. In such strong interaction zones, dissipation of energy in a turbulent cascade is important (e.g. Guillard et al. 2010). The eastern terminal lobe of the jet is, for example, the working surface of the jet as it propagates outwards, which exactly the region where you would expect strong dissipation of the bulk kinetic energy of the jet.

The molecular gas likely formed in situ in the post-shock gas, which has important implications for the state of the circum-galactic halo and cluster gas. Recent observations of the Spiderweb galaxy using ATCA discovered a large region of $\mathrm{CO}(1-0)$ surrounding the $\mathrm{H}_{z} \mathrm{RG}$ with strong emission near some of the radio knots and lobes, which suggests that the passage of the radio jet enhances the molecular column densities (Emonts et al., in prep.). Finding strong $\mathrm{H}_{2} \mathrm{O}$ line emission near the radio jet provides evidence of the formation of molecular gas in the post-shocked gas of the expanding radio source (see Guillard et al. 2010, 2012; Appleton et al. 2013, for a discussion of this mechanism in the violent collision in the Stephan's Quintet group). This is also substantiated by the low relative velocities of the $\mathrm{H}_{2} \mathrm{O}$ emission, which are consistent with the low velocities observed in the extended diffuse CO emission. Such a mechanism might also explain the distribution of the $\mathrm{CO}$ emission observed in local clusters (Salomé et al. 2011) and around other $\mathrm{H}_{z} \mathrm{RGs}$ (Emonts et al. 2014, and references therein). Finding $\mathrm{H}_{2} \mathrm{O}$ emission that indicates strong dissipation of the bulk kinetic energy of expanding radio jets provides a direct link between the jets and the formation of molecular gas and stars in the halo of the radio galaxy.

\subsection{Mass estimates}

As argued above, the [CI]2-1 line is a better tracer of the cold molecular gas than the high-J CO lines, owing to their lower critical density. We therefore use the $[\mathrm{CI}] 2-1$ to estimate the total molecular gas mass, following the approach of Papadopoulos et al. (2004) and Alaghband-Zadeh et al. (2013):

$$
\begin{aligned}
M_{\mathrm{H}_{2}}= & 1375.8 D_{\mathrm{L}}^{2}(1+z)^{-1}\left[\frac{X_{[\mathrm{CI}]}}{10^{-5}}\right]^{-1} \\
& \times\left[\frac{A_{21}}{10^{-7} \mathrm{~s}^{-1}}\right]^{-1} Q_{21}^{-1}\left[\frac{S_{[\mathrm{CI}] 2-1} \mathrm{~d} V}{\mathrm{Jy} \mathrm{km} \mathrm{s}^{-1}}\right],
\end{aligned}
$$

where $X_{[\mathrm{CI}]}$ is the [CI]-to- $\mathrm{H}_{2}$ abundance for which we adopt the literature-standard of $3 \times 10^{-5}, Q_{21}$ is the density dependent excitation factor, for which we assume the median value of 0.57 (see
Papadopoulos et al. 2004), and $A_{21}$ is the Einstein A coefficient of $2.68 \times 10^{-7} \mathrm{~s}^{-1}$. We find a total $\mathrm{H}_{2}$ molecular gas mass for both components of $3.3 \times 10^{10} M_{\odot}$. We note that this value depends on the adopted $Q_{21}$ : for the highest $Q_{21}=1.0$, the $M\left(\mathrm{H}_{2}\right)$ drops to $2.2 \times 10^{10} M_{\odot}$, while for the lowest $Q_{21}=0.15$, the $M\left(\mathrm{H}_{2}\right)$ is $1.5 \times 10^{11} M_{\odot}$ The median value is close to $M\left(\mathrm{H}_{2}\right)=6 \times 10^{10} M_{\odot}$ that is derived from $\mathrm{CO}(1-0)$ (Emonts et al. 2013). This total mass of diffuse molecular gas, as probed by the [CI]2-1 emission, is clearly higher than the warm $(T>300 \mathrm{~K})$ molecular gas mass of order $10^{7}$ to $10^{9} M_{\odot}$ (Ogle et al. 2012), which suggests an order of magnitude ratio of warm-to-cold molecular gas between $0.1-10 \%$. Ogle et al. (2012) suggest that the gas is heated by the radio jet from the AGN interacting with the ambient ISM of the $\mathrm{H}_{z} \mathrm{RG}$. Furthermore, Nesvadba et al. (2006) find a mass of ionised gas of $\sim 3 \times 10^{9} M_{\odot}$. The gas masses of various components of the ISM of the $\mathrm{H}_{z} \mathrm{RG}$ are up to one order of magnitude lower than the stellar mass of $<1.8 \times 10^{12} M_{\odot}$ (De Breuck et al. 2010), suggesting that this is already quite an evolved galaxy.

For component 2, the $\mathrm{H}_{2}$ gas mass of the broad component is $1.6 \times 10^{10} M_{\odot}$. We use this to constrain the size of this region by knowing that the molecular mass has to be smaller than the dynamical mass $\left(M\left(\mathrm{H}_{2}\right)<M_{\mathrm{dyn}}=R \cdot v^{2} / G\right)$, and by using the velocity from the FWHM (see Table 2), we find that $R<60 \mathrm{pc}$. Though uncertain, this region has a size comparable to those of the obscuring torus and the inner narrow line regions of QSOs, thus suggesting the presence of an additional radio-quiet AGN merging with the radio-loud AGN in component 1 (Emonts et al. 2015). Applying the same methods to component 1 , which has $\mathrm{a} \mathrm{H}_{2}$ gas mass of $1.7 \times 10^{10}$, puts an upper limit of $1 \mathrm{kpc}$ on the size of the emitting region.

To determine the dust mass, we need to integrate over the full SED of the dust emission. As we only have a single observation at $246 \mathrm{GHz}$, we fix the shape of the SED by assuming a greybody that is characterised by $\beta=1.5$ and $T_{\mathrm{d}}=40 \mathrm{~K}$. Following the approach of De Breuck et al. (2003), using

$$
M_{\mathrm{d}}=\frac{S_{\mathrm{obs}} D_{\mathrm{L}}^{2}}{(1+z) \kappa_{\mathrm{d}}\left(v_{\text {rest }}\right) B\left(v_{\text {rest }}, T_{\mathrm{d}}\right)},
$$

where $S_{\text {obs }}$ is the flux density at the observed frequency, $D_{\mathrm{L}}$ is the luminosity distance, $\kappa_{\mathrm{d}}\left(v_{\text {rest }}\right)$ is the rest-frequency mass absorption coefficient, which we here calculate by scaling the $850 \mu \mathrm{m}$ absorption coefficient of $0.076 \mathrm{~m}^{2} \mathrm{~kg}^{-1}$ from Stevens et al. (2003) to $246 \mathrm{GHz}$, and $B\left(v_{\text {rest }}, T_{\mathrm{d}}\right)$ is the Planck function for isothermal dust grain emission at a dust temperature $T_{\mathrm{d}}$ (De Breuck et al. 2003), we derive a dust mass of $M_{\mathrm{d}}=$ $6.1 \times 10^{8} M_{\odot}$ for the $\mathrm{Hz} \mathrm{RG}$. This is marginally higher than what was estimated by Stevens et al. 2003 of $4.6 \times 10^{8} M_{\odot}$ using the $850 \mu$ m flux peak flux.

Taking the same approach and assumed dust temperature, we find the dust masses of the two companions \#8 and \#10 (see Table 4).

\subsection{Star formation rate}

Using Spitzer and Herschel PACS+SPIRE photometry, Seymour et al. (2012) show that the strong AGN and vigorous star formation contribute with roughly equal amounts to the IR luminosity in the Spiderweb galaxy. While the AGN component dominates at the high frequency end of the thermal dust emission, the star formation is expected to contribute $>90 \%$ of the flux at the observed (comparatively low) frequencies of our ALMA data. However, the flux spectral index of the $\mathrm{H} z \mathrm{RG}$ and companion \#10, implies that the flux at these wavelength are likely to 
Table 4. Estimated dust masses, FIR luminosities and SFRs for the $\mathrm{H} z \mathrm{RG}$ and companions using the measured fluxes listed in Table 1 and Eqs. (1)-(5).

\begin{tabular}{lccc}
\hline \hline Component & $\begin{array}{c}M_{\mathrm{d}} \\
{\left[M_{\odot}\right]}\end{array}$ & $\begin{array}{c}L_{\mathrm{FIR}} \\
{\left[L_{\odot}\right]}\end{array}$ & $\begin{array}{c}S F R \\
{\left[M_{\odot} \mathrm{yr}^{-1}\right]}\end{array}$ \\
\hline $\mathrm{HzRG}$ & $<6.1 \times 10^{8}$ & $<4.4 \times 10^{12}$ & $<440$ \\
\hline Companion \#8 & $<8.5 \times 10^{7}$ & $<6.2 \times 10^{11}$ & $<62$ \\
Companion \#10 & $<6.5 \times 10^{7}$ & $<4.7 \times 10^{11}$ & $<47$ \\
\hline Sum & - & $<5.4 \times 10^{12}$ & $<549$ \\
\hline Water (west) & $<7.0 \times 10^{7}$ & $<5.7 \times 10^{11}$ & $<57$ \\
Water (east) & $<7.0 \times 10^{7}$ & $<5.7 \times 10^{11}$ & $<57$ \\
\hline
\end{tabular}

Notes. The dust masses, FIR luminosities and SFRs are all upper limits, since the $\mathrm{H} z \mathrm{RG}$ and companion \#10 have a contribution from synchrotron emission and companion \#8 is a tentative detection.

be contaminated by synchrotron emission. We can therefore use our continuum flux to determine upper limits on the SFR in the host galaxy and the companions.

We estimate the SFR of the $\mathrm{H}_{z} \mathrm{RG}$ and companions by fixing the dust temperature and emissivity index (as for the dust masses) and calculating $L_{\mathrm{FIR}}$ using

$L_{\mathrm{FIR}}=4 \pi M_{\mathrm{d}} \int_{0}^{\infty} \kappa_{\mathrm{d}}\left(v_{\text {rest }}\right) B\left(v_{\text {rest }}, T_{\mathrm{d}}\right) \mathrm{d} v$,

which yields

$L_{\mathrm{FIR}}=4 \pi \Gamma[\beta+4] \zeta[\beta+4] D_{\mathrm{L}}^{2} x^{-(\beta+4)}\left(\mathrm{e}^{x}-1\right) S_{\mathrm{obs}} v_{\mathrm{obs}}$,

where $\Gamma$ and $\zeta$ are the Gamma and Riemann $\zeta$ functions and $x=h v_{\text {rest }} / k T_{\mathrm{d}}$ (De Breuck et al. 2003). Again, assuming $\beta=1.5$ and $T_{\mathrm{d}}=40 \mathrm{~K}$, we find the $L_{\mathrm{FIR}}<5.1 \times 10^{12} L_{\odot}$ for the $\mathrm{H}_{z} \mathrm{RG}$, and $<7.2 \times 10^{11} L_{\odot}$ for companion \#8 and $<5.4 \times 10^{11} L_{\odot}$ for companion \#10 (see Table 4).

Using

$S F R=\delta_{\mathrm{MF}} \delta_{\mathrm{SB}}\left(L_{\mathrm{FIR}} / 10^{10} L_{\odot}\right) M_{\odot} \mathrm{yr}^{-1}$,

where $\delta_{\mathrm{MF}}$ is the stellar mass function and $\delta_{\mathrm{SB}}$ is the fraction of FIR emission that is heated by the starburst, which we assume to be unity at these long wavelengths, the $L_{\mathrm{FIR}}$ can be converted to the SFR. We assume $\delta_{\mathrm{MF}}=1$ and derive the SFRs of $<440 M_{\odot} \mathrm{yr}^{-1}$ for the $\mathrm{Hz} \mathrm{RG},<62 M_{\odot} \mathrm{yr}^{-1}$ for companion \#8 and $<47 M_{\odot} \mathrm{yr}^{-1}$ for companion \#10 (see Table 4 ). The sum of the estimated FIR luminosity of the $\mathrm{HzRG}$ and the two companion sources is higher than the total starburst-heated IR luminosity of $5.6 \times 10^{12} L_{\odot}$ of Drouart et al. 2014 , less than the $7.9 \times 10^{12} L_{\odot}$ of Seymour et al. (2012). We warn that our approximation of the SED with a single greybody function with an assumed emissivity index is rather crude; moreover the $246 \mathrm{GHz}$ emission from the $\mathrm{H}_{z} \mathrm{RG}$ and companion \#10 may also contain a contribution from synchrotron emission, which would over-estimate the SFR.

\subsubsection{SFR of the Spiderweb galaxy and its companions}

Kuiper et al. (2011) find a bi-model velocity distribution of galaxies close in projection to the Spiderweb galaxy, which suggests two groups (or regions) of galaxies flowing into the circumgalactic environment at high velocity $\left(\sim 400\right.$ to $\left.1600 \mathrm{~km} \mathrm{~s}^{-1}\right)$. Comparing the velocity and spatial distribution of the galaxies with that found in massive halos at $z \sim 2$ within the Millennium simulation, they conclude that the Spiderweb galaxy is best described by the merger of two galaxy groups or flows of galaxies into the over-dense environment of the powerful $\mathrm{HzRG}$.

The SFRs that we determine here for the $\mathrm{H}_{z} \mathrm{RG}$ and companion \#10 and \#8 are 10-50 times higher than determined by Hatch et al. (2009) using optical HST imaging. However, the continuum emission in both the $\mathrm{HzRG}$ and companion \#10 are contaminated by synchrotron emission. For the $\mathrm{H}_{z} \mathrm{RG}$ there are two components and only one of them, the AGN core, is contaminated up to $\sim 50 \%$ (see Sect. 3.1 ). The fact that the continuum emission is extended in the direction that connects these two components (see Fig. 1) suggests that both must have roughly similar $246 \mathrm{GHz}$ flux densities. The synchrotron contamination for the combined Spiderweb galaxy is therefore estimated to be no more than $\sim 25 \%$. For companion \#10, all of the emission could be due to synchrotron (see Sect. 3.1). Observations of the synchrotron spectrum around $\sim 100 \mathrm{GHz}$ are needed to determine if it remains straight out to $246 \mathrm{GHz}$, or if there is a downturn owing to energy losses of the most energetic electrons. In the latter case, even companion \#10 could still be dominated by star formation. In this extreme case, then its SFR and that of companion \#8 would suggest that (over the scale of our ALMA data) about $\sim 20 \%$ of the star formation is taking place outside the $\mathrm{H}_{z} \mathrm{RG}$ in companion sources. We note that companion \#8 is a marginal $3 \sigma$ detection and that the two additional companions (\#12 and \#17) show hints of $246 \mathrm{GHz}$ emission at $\sim 1.5 \sigma$ significance. To confirm if these hints of emission are real requires deeper $246 \mathrm{GHz}$ observations. For the remaining sources in the surroundings of the Spiderweb galaxy, the sensitivity of our data is too low to detect them, meaning that their SFR is $\lesssim 50 M_{\odot} \mathrm{yr}^{-1}$.

\section{Conclusions}

We have observed the $\mathrm{H} z \mathrm{RG}$ MRC1138-262 (the Spiderweb galaxy) with ALMA Band 6 in Cycle 1 for 49 min on-source.

- We detect strong $246 \mathrm{GHz}$ continuum emission at the position of the $\mathrm{HzRG}$, weaker $246 \mathrm{GHz}$ continuum emission for a companion to the west, and tentative emission to the north. Companion \#10 of Kuiper et al. (2011) at $z=2.1446$ is the brightest of the two companions and is detected in $\mathrm{CO}(7-6)$, while $[\mathrm{CI}] 2-1$ is shifted out of the observed band. The other companion is too weak to be detected in line emission.

- The flat spectral indexes of the $\mathrm{HzRG}$ and companion \#10 suggest a contribution to the $246 \mathrm{GHz}$ continuum emission from synchrotron emission. This means that the FIR luminosities and SFRs of these two components are upper limits.

- We detect strong [CI]2-1 emission in two components: one at the position of the $\mathrm{H}_{z} \mathrm{RG}$, and one with a small offset of $0 \prime .5$ to the south-east from the central position. The $[\mathrm{CI}] 2-1$ emitting components show significantly different velocity profiles: component 1 shows a Lorentzian profile, and component 2 is a double Gaussian with velocity widths of 250 and $1100 \mathrm{~km} \mathrm{~s}^{-1}$.

- Using the molecular gas-mass estimate of the broad [CI]2-1 of component 2, we put an upper limit on the size of the emitting region of $60 \mathrm{pc}$. This size suggests the possible existence of an additional radio quiet AGN, merging with the radio loud $\mathrm{AGN}$ in component 1.

- The $\mathrm{CO}(7-6) /[\mathrm{CI}] 2-1$ line ratio for the Spiderweb galaxy is lower than that of a comparison sample. This ratio can be due to a relatively low excitation molecular gas reservoir and/or a strong cosmic ray field which, preferentially, destroys CO. As the comparison sample is composed of only 
lensed sources from the literature, differential lensing may also have an influence, where the dense $\mathrm{CO}(7-6)$ gas in the comparison sample is amplified more than the more diffuse [CI]2-1 gas.

- We detect $\sim 4 \sigma \mathrm{H}_{2} \mathrm{O}$ emission lines at two positions: to the west at knot B4 (at the bend) in the radio jet and to the east, upstream of knot A. No $246 \mathrm{GHz}$ continuum emission is detected at the position of the $\mathrm{H}_{2} \mathrm{O}$ emission lines, meaning that heating from IR emission cannot be the source of the $\mathrm{H}_{2} \mathrm{O}$ emission. The positions (upstream of the two knots in the radio jet), the lack of $246 \mathrm{GHz}$ continuum emission at these positions and the small offset of the line centres, are consistent with heating by slow shocks in dense molecular gas. The relatively bright $\mathrm{H}_{2} \mathrm{O}$ emission indicates strong dissipation of the energy of the expanding jets in the halo of MRC1138-262.

Multiple studies have established that the Spiderweb galaxy is a system of merging galaxies, and the likely progenitor of a $\mathrm{cD}$ galaxy. Our water detections upstream of the main radio hotspots show that the radio jets can cause the gas in the ISM and inter-cluster gas to cool, increasing the fraction of extended molecular gas (Emonts et al., in prep.). The formation of this molecular reservoir likely supports the formation of the young stars that are observed in the halo of MRC1138-262. The much brighter than expected [CI]2-1 emission indicates the power of this line to study the detailed distribution of the molecular gas reservoir in this intriguing source, and possibly other $\mathrm{H}_{z} \mathrm{RGs}$.

Acknowledgements. This paper makes use of the following ALMA data ADS/JAO.ALMA\#2012.1.01087.S. ALMA is a partnership of ESO (representing its member states), NSF (USA) and NINS (Japan), together with NRC (Canada), NSC and ASIAA (Taiwan), and KASI (Republic of Korea), in cooperation with the Republic of Chile. The Joint ALMA Observatory is operated by ESO, AUI/NRAO, and NAOJ. B.G. acknowledges support from the ERC Advanced Investigator programme DUSTYGAL 321334. B.E. acknowledges funding through the European Union FP7-PEOPLE-2013-IEF grand 62435.

\section{References}

Alaghband-Zadeh, S., Chapman, S. C., Swinbank, A. M., et al. 2013, MNRAS, 435,1493

Alloin, D., Guilloteau, S., Barvainis, R., Antonucci, R., \& Tacconi, L. 1997, A\&A, 321, 24

Appleton, P. N., Guillard, P., Boulanger, F., et al. 2013, ApJ, 777, 66

Bicknell, G. V., Dopita, M. A., Tsvetanov, Z. I., \& Sutherland, R. S. 1998, ApJ 495, 680

Bisbas, T. G., Papadopoulos, P. P., \& Viti, S. 2015, ApJ, 803, 37
Carilli, C. L., Röttgering, H. J. A., van Ojik, R., et al. 1997, ApJS, 109, 1 Carilli, C. L., Harris, D. E., Pentericci, L., et al. 2002, ApJ, 567, 781 Cox, P., Krips, M., Neri, R., et al. 2011, ApJ, 740, 63 Danielson, A. L. R., Swinbank, A. M., Smail, I., et al. 2011, MNRAS, 410, 1687 Dannerbauer, H., Kurk, J. D., De Breuck, C., et al. 2014, A\&A, 570, A55

De Breuck, C., Neri, R., Morganti, R., et al. 2003, A\&A, 401, 911 De Breuck, C., Seymour, N., Stern, D., et al. 2010, ApJ, 725, 36 Downes, D., \& Solomon, P. M. 2003, ApJ, 582, 37

Drouart, G., De Breuck, C., Vernet, J., et al. 2014, A\&A, 566, A53 Emonts, B. H. C., Feain, I., Röttgering, H. J. A., et al. 2013, MNRAS, 430, 3465 Emonts, B. H. C., Norris, R. P., Feain, I., et al. 2014, MNRAS, 438, 2898 Emonts, B. H. C., De Breuck, C., Lehnert, M. D., et al. 2015, A\&A, 584, A99 Ferkinhoff, C., Brisbin, D., Parshley, S., et al. 2014, ApJ, 780, 142 Flower, D. R., \& Pineau Des Forêts, G. 2010, MNRAS, 406, 1745 Glover, S. C. O., Clark, P. C., Micic, M., \& Molina, F. 2015, MNRAS, 448, 1607

Goicoechea, J. R., Chavarría, L., Cernicharo, J., et al. 2015, ApJ, 799, 102 Greve, T. R., Ivison, R. J., \& Stevens, J. A. 2006, Astron. Nachr., 327, 208 Guillard, P., Boulanger, F., Pineau Des Forêts, G., \& Appleton, P. N. 2009, A\&A, 502,515

Guillard, P., Boulanger, F., Cluver, M. E., et al. 2010, A\&A, 518, A59

Guillard, P., Boulanger, F., Pineau des Forêts, G., et al. 2012, ApJ, 749, 158

Hatch, N. A., Overzier, R. A., Röttgering, H. J. A., Kurk, J. D., \& Miley, G. K. 2008, MNRAS, 383, 931

Hatch, N. A., Overzier, R. A., Kurk, J. D., et al. 2009, MNRAS, 395, 114 Humphrey, A., Villar-Martín, M., Vernet, J., et al. 2008, MNRAS, 383, 11 Kneib, J.-P., Neri, R., Smail, I., et al. 2005, A\&A, 434, 819

Kuiper, E., Hatch, N. A., Miley, G. K., et al. 2011, MNRAS, 415, 2245

Kurk, J. D., Pentericci, L., Overzier, R. A., Röttgering, H. J. A., \& Miley, G. K. 2004a, A\&A, 428, 817

Kurk, J. D., Pentericci, L., Röttgering, H. J. A., \& Miley, G. K. 2004b, A\&A, 428, 793

Lestrade, J.-F., Combes, F., Salomé, P., et al. 2010, A\&A, 522, L4

Lonsdale, C. J., \& Barthel, P. D. 1986, AJ, 92, 12

Lupu, R. E., Scott, K. S., Aguirre, J. E., et al. 2012, ApJ, 757, 135

Miley, G. K., Overzier, R. A., Zirm, A. W., et al. 2006, ApJ, 650, L29

Nesvadba, N. P. H., Lehnert, M. D., Eisenhauer, F., et al. 2006, ApJ, 650, 693

Ogle, P., Davies, J. E., Appleton, P. N., et al. 2012, ApJ, 751, 13

Omont, A., Yang, C., Cox, P., et al. 2013, A\&A, 551, A115

Papadopoulos, P. P., Thi, W.-F., \& Viti, S. 2004, MNRAS, 351, 147

Pentericci, L., Röttgering, H. J. A., Miley, G. K., Carilli, C. L., \& McCarthy, P. 1997, A\&A, 326, 580

Pentericci, L., Röttgering, H. J. A., Miley, G. K., et al. 1998, ApJ, 504, 139

Pentericci, L., Kurk, J. D., Röttgering, H. J. A., et al. 2000, A\&A, 361, L25

Pentericci, L., Kurk, J. D., Carilli, C. L., et al. 2002, A\&A, 396, 109

Riechers, D. A., Bradford, C. M., Clements, D. L., et al. 2013, Nature, 496, 329

Röttgering, H. J. A., van Ojik, R., Miley, G. K., et al. 1997, A\&A, 326, 505

Salomé, P., Combes, F., Revaz, Y., et al. 2011, A\&A, 531, A85

Serjeant, S. 2012, MNRAS, 424, 2429

Seymour, N., Altieri, B., De Breuck, C., et al. 2012, ApJ, 755, 146

Stevens, J. A., Ivison, R. J., Dunlop, J. S., et al. 2003, Nature, 425, 264

van der Werf, P. P., Berciano Alba, A., Spaans, M., et al. 2011, ApJ, 741, L38

Walter, F., Weiß, A., Downes, D., Decarli, R., \& Henkel, C. 2011, ApJ, 730, 18

Weiß, A., Henkel, C., Downes, D., \& Walter, F. 2003, A\&A, 409, L41

Yang, C., Gao, Y., Omont, A., et al. 2013, ApJ, 771, L24 